\title{
Growth on a Finite Planet: Resources, Technology, and Population in the Long Run
}

\author{
Pietro Peretto Simone Valente \\ Duke University NTNU Trondheim
}

April 17, 2013

ERID Working Paper Number 147

This paper can be downloaded without charge from the Social Science Research Network Electronic Paper Collection:

http://ssrn.com/abstract=2275338

\section{Economic Research Initiatives at Duke WORKING PAPERS SERIES

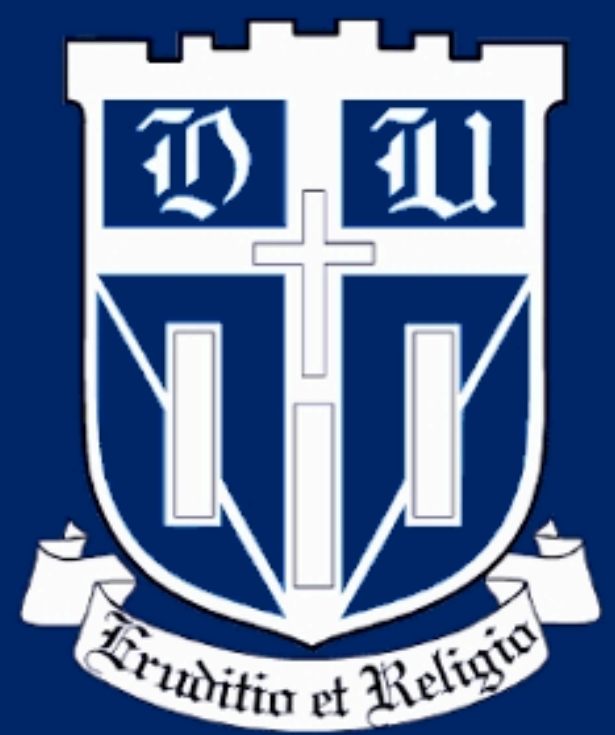




\title{
Growth on a Finite Planet: Resources, Technology and Population in the Long Run
}

\author{
Pietro F. Peretto, Duke University* \\ Simone Valente, NTNU Trondheim ${ }^{\dagger}$
}

April 17, 2013

\begin{abstract}
We study the interactions between technological change, resource scarcity and population dynamics in a Schumpeterian model with endogenous fertility. We find a pseudo-Malthusian equilibrium in which population is constant and determined by resource scarcity while income grows exponentially. If labor and resources are substitutes in production, income and fertility dynamics are selfbalancing and the pseudo-Malthusian equilibrium is the global attractor of the system. If labor and resources are complements, income and fertility dynamics are self-reinforcing and drive the economy towards either demographic explosion or collapse. Introducing a minimum resource requirement per capita, we obtain constant population even under complementarity.
\end{abstract}

Keywords Endogenous Innovation, Resource Scarcity, Population Growth, Fertility Choices

JEL codes E10, L16, O31, O40

*Pietro F. Peretto, Room 241, Department of Economics, Duke University, Durham, NC 27708 (USA). Phone: (919) 660-1807. Fax: (919) 684-8974. E-mail: peretto@econ.duke.edu.

†Simone Valente, Department of Economics, NTNU, 7491 Trondheim, Norway. Phone: +4773591932. E-mail: simone.valente@svt.ntnu.no. 


\section{Introduction}

More than two centuries after the publication of Thomas Malthus' (1798) Essay on the Principle of Population, understanding the interactions between economic growth, resource scarcity and population remains a central aim of scholars in different fields of social sciences. The debate revolves around two fundamental questions: whether a larger population is good or bad for human development and welfare (Birdsall and Sinding, 2001; Kelley, 2001), and how population growth changes with economic conditions (Kremer, 1993, Wang et al., 1994). In the past century, economists treated these issues as distinct subjects, casting the first problem in the realm of welfare/resource economics (Robinson and Srinivasian, 1997) and addressing the second in the context of fertility theories (Nerlove and Raut, 1997). It is increasingly evident, however, that little progress can be made without tackling both issues at the same time: assessing the economic consequences of a growing population requires considering the feedback effects of tighter resource scarcity on fertility (Bloom and Canning, 2001).

This recognition underlies two strands of recent literature. The first is Unified Growth Theory (UGT), a framework that provides consistent explanations of the historical phases of development, from the Malthusian Stagnation to the current regime of sustained growth in per capita incomes (Galor and Weil, 2000; Galor, 2005, 2011). The two building blocks of UGT models are endogenous fertility and the assumption that consumption goods are produced by means of human capital and a natural resource, typically land. This structure highlights the central mechanism behind economy-environment interactions: population growth affects natural resource scarcity and labor productivity, while income dynamics induce feedback effects on fertility that determine future population growth.

Population-resource interactions are also studied in the literature on bio-economic systems, which seeks to explain the rise and fall of civilizations. These contributions draw explicit links between population dynamics and the laws of biological regeneration that govern resource availability: individuals operate in a closed system - e.g., islands - and the resource stock follows a logistic process that is directly affected by harvesting choices. The interaction between population growth and biological laws generates rich dynamics, including feast-famine equilibrium paths and/or envi- 
ronmental crises that can eventually drive the human society to extinction (Taylor, 2009). Bio-economic models have been calibrated to replicate the collapse of Easter Island and similar historical episodes (Brander and Taylor, 1998; Basener and Ross, 2005; Good and Reuveny, 2009). Several authors have argued that the Easter Island economy is a metaphor of resource-based closed systems like Planet Earth and extended the model to include manufactured goods (Reuveny and Decker, 2000), intentional capital bequests (Harford, 2000) and endogenous technological change (Dalton et al., 2005).

In this paper, we take a different perspective and investigate the mechanism linking resource scarcity, incomes and population in a Schumpeterian model of endogenous growth. Unlike UGT, our analysis does not seek to explain the transition from the Malthusian Stagnation to modern growth regimes, but aims to build a theory of economy-environment interactions capable of addressing one of the main future challenges for modern industrialized economies: how to sustain income growth in a finite habitat. In answering this question, standard balanced growth models are not satisfactory: their prediction of exponential population growth is clearly at odds with the fact that Planet Earth has a finite carrying capacity of people. We tackle this issue by studying whether and under what circumstances populationresource interactions generate long-run equilibria where income grows at constant (endogenous) rates while population achieves a constant (endogenous) level. The existence of such equilibria requires that long-term economic growth be driven by the accumulation of intangible assets. Accordingly, the modern theory of R\&D-based productivity growth is the natural starting point.

In our model, firms produce different varieties of manufacturing goods by means of labor and a resource in fixed aggregate supply - e.g., land - and household utility maximization determines fertility choice. The central insight of our analysis is that as population growth raises natural scarcity, the strength of the resource price response determines a resource income effect that drives the feedback response of fertility and thereby the qualitative dynamics of population. The literature generally neglects these price effects because the existing models either abstract from the resource market (e.g., Galor and Weil, 2000) or, when they allow for a resource market, assume a unit elasticity of substitution between labor and resources (e.g., 
Lucas, 2002). ${ }^{1}$ In our model, instead, we consider a generic production function displaying constant returns to scale and show that the effect of the resource price on fertility drives the economy towards different long-run equilibria depending on whether labor and resources are complements or substitutes. Before describing our results in detail, we emphasize another characteristic of our analysis.

We employ a Schumpeterian model of endogenous growth in which horizontal and vertical innovations coexist: manufacturing firms undertake $R \& D$ to increase their total factor productivity while outside entrepreneurs design new products and set up new firms in order to serve the market (Peretto, 1998; Dinopoulos and Thompson, 1998). This class of models has received substantial empirical support in recent years (Laincz and Peretto, 2006; Ha and Howitt, 2007; Madsen, 2008; Madsen et al., 2010; Madsen and Ang, 2011) and is particularly useful in addressing our research question because it predicts that the effect of endowments on growth is only temporary. Specifically, product proliferation, i.e., net entry, sterilizes the (strong) scale effect in the long run because it fragments the aggregate market into submarkets whose size does not increase with the size of the endowments. In our analysis with endogenous fertility, the elimination of the (strong) scale effect generates long-run equilibria in which population is constant and does not affect productivity growth.

Our first result is that there exists a pseudo-Malthusian steady state in which income per capita grows at a constant rate and population is constant. We label this steady state pseudo-Malthusian because the equilibrium population level is proportional to the resource endowment but is not constrained by technology. Importantly, the existence of the pseudo-Malthusian equilibrium is not due to ad-hoc assumptions on preferences for fertility or the reproduction technology - in fact, we posit a very standard specification of the costs and benefits of reproduction - but is exclusively determined by the price effects arising under complementarity or substitutability. Indeed, if we impose unit elasticity of input substitution, the pseudo-Malthusian steady state disappears and the equilibrium path displays exponential population growth as in most balanced growth models.

\footnotetext{
${ }^{1}$ An exception is Bretschger (2011), who considers poor substitution (complementarity) between labor and an exhaustible resource in a Romer-style model of endogenous growth that exhibits the strong scale effect.
} 
Our second result concerns how the elasticity of input substitution determines the stability properties of the equilibrium. If labor and resources are substitutes, the pseudo-Malthusian steady state is a global attractor, and represents the longrun equilibrium of the economy for any initial condition, because the feedback effect of scarcity on fertility is self-balancing. Population growth reduces the resourcelabor ratio but, due to substitutability, the resource price rises moderately. As a consequence, resource income declines over time and the fertility rate decreases until population reaches a constant equilibrium level. If labor and resources are complements, instead, the pseudo-Malthusian steady state is a separating threshold: if the resource is initially scarce (abundant), population follows a diverging path implying demographic explosion (collapse). The reason is that complementarity generates a self-reinforcing feedback effect: starting from the pseudo-Malthusian steady state, a rise in population yields a resource price increase that raises resource income, boosting fertility and thereby population growth. Symmetrically, if the deviation from the pseudo-Malthusian steady state is toward resource abundance i.e., a drop in population - the resource price response induces lower income and further population decline.

Third, we extend the analysis to consider a more general environment in which the input elasticity of substitution varies with the resource price: the economy may transit from a region of complementarity to a region of substitutability and viceversa. In this case, there are two steady states with distinct basins of attraction and the long-run equilibrium becomes state dependent, in the sense that the economy may converge towards the pseudo-Malthusian steady state or diverge from it depending on the initial level of the resource endowment.

Our fourth result is that, if we extend the model to introduce a minimum resource requirement per adult - e.g., residential land - the economy may avoid demographic explosion even under complementarity. As households respond to a price signal that reflects congestion, fertility rates are subject to an enhanced preventive check that always stabilizes the population level as it grows too large. Since this mechanism also operates with Cobb-Douglas technology, the model shows that the standard result of exponential population growth often found in the literature is a rather special case: if the net resource supply per capita is subject to a lower bound, the economy converges towards a pseudo-Malthusian equilibrium even though labor and 
resources are neither complements nor substitutes.

\section{The model}

A representative household purchases differentiated consumption goods and chooses the number of children in order to maximize utility. The household supplies labor services and a natural resource (e.g., land) in competitive markets, and accumulates wealth in the form of financial assets. Each variety of consumption good is supplied by one monopolistic firm and productivity growth stems from two types of innovations. First, the mass of manufacturing firms increases over time due to the development of new product lines (horizontal innovation). Second, each firm undertakes in-house R\&D to increase it own productivity (vertical innovation). The interplay between horizontal and vertical innovations allows the economy to grow in the long run at a constant endogenous rate that is independent of factor endowments (Peretto, 1998; Dinopoulos and Thompson, 1998; Peretto and Connolly 2007). This class of models is receiving strong empirical support (Laincz and Peretto, 2006; Ha and Howitt, 2007; Madsen, 2008; Madsen et al. 2010; Madsen and Ang, 2011), which further legitimates its use in our theoretical analysis. Connolly and Peretto (2003) studied the role of endogenous fertility in this framework. Our analysis extends the model to include privately-owned natural resources and varying degrees of substitutability between labor and resource inputs, exploiting the tractable framework developed by Peretto (2011). ${ }^{2}$

\section{$2.1 \quad$ Households}

The representative household maximizes present-value welfare at time $t$,

$$
U(t)=\int_{t}^{\infty} e^{-\rho(v-t)} \log u(v) d v
$$

where $\log u(v)$ is the instantaneous utility of each adult at time $v$, and $\rho>0$ is the discount rate. We specify preferences according to the Barro-Becker approach to fertility choice in continuous time (see Barro and Sala-i-Martin, 2004, pp. 411-421).

\footnotetext{
${ }^{2}$ Peretto (2011) studies the effects on income, growth and welfare of a shock to the natural resource endowment in a model with constant population.
} 
Instantaneous utility depends on individual consumption, the fertility rate - defined as the mass of children per adult and denoted by $b$ - and population size:

$$
\log u(t)=\log \left[\int_{0}^{N(t)}\left(X_{i}(t) / L(t)\right)^{\frac{\epsilon-1}{\epsilon}} d i\right]^{\frac{\epsilon}{\epsilon-1}}+\mu \log b(t)+(\sigma+1) \log L(t),
$$

where $N$ is the mass of consumption goods, $X_{i}$ is aggregate consumption of the $i$-th good, $L$ is the mass of adults, $\epsilon>1$ is the elasticity of substitution among differentiated goods, $\mu>0$ is the elasticity of utility with respect to individual fertility and $\sigma>0$ is the net elasticity of utility with respect to adult family size. ${ }^{3}$ The law of motion of adult population is

$$
\dot{L}(t)=L(t)(b(t)-d),
$$

where $d>0$ is the death rate, assumed exogenous and constant.

Each adult is endowed with one unit of time, which can be spent either working or rearing children. Denoting the time cost of child rearing by $\psi$, each adult supplies $1-\psi b(t)$ units of labor. In addition, the household supplies inelastically $R$ units of a non-exhaustible natural resource (e.g., land) to manufacturing firms. The wealth constraint thus reads

$$
\dot{A}(t)=r(t) A(t)+w(t) L(t)(1-\psi b(t))+p(t) R(t)-Y(t),
$$

where $A$ is assets holding, $r$ is the rate of return on assets, $w$ is the wage rate, $L(1-\psi b)$ is total labor supply, $p$ is the market price of the resource, and $Y$ is consumption expenditure. The household solves the problem in two steps. First, it chooses the quantity of each consumption good in order to maximize the instantaneous utility (2) subject to the expenditure constraint

$$
Y(t)=\int_{0}^{N(t)} P_{i}(t) X_{i}(t) d i
$$

where $P_{i}$ is the price of the $i$-th good. In the second step, the household chooses the time paths of expenditure and the fertility rate in order to maximize intertemporal welfare (1) subject to the demographic law (3) and the wealth constraint (4).

\footnotetext{
${ }^{3}$ Specification (2) is the continuous-time equivalent of specification [9.52] in Barro and Salai-Martin (2004, p. 410). As shown in the Appendix, the consumption term reduces to $\log (Y(t) / L(t))$, where $Y(t)$ is total consumption expenditure, so that the net elasticity of utility to population size reduces to $\sigma+1-1=\sigma$.
} 


\subsection{Production and Vertical Innovation}

Each consumption good is produced by a single monopolist that makes two decisions. First, it chooses the cost-minimizing combination of production inputs at each instant. Second, it chooses the time path of $R \& D$ effort in order to maximize the present value of its future profits.

Specifically, firm $i$ operates the production technology

$$
X_{i}=Z_{i}^{\theta} \cdot F\left(L_{X_{i}}-\phi, R_{i}\right),
$$

where $X_{i}$ is output, $L_{X_{i}}$ is labor employed in production, $\phi>0$ is a fixed labor cost, $R_{i}$ is the resource input and $F(\cdot, \cdot)$ is a standard production function homogeneous of degree one in its arguments that may exhibit elasticity of input substitution below or above unity. Whether labor and the resource are complements or substitutes matters for our results and we will discuss all possible scenarios, including the case of unit elasticity where $F(\cdot, \cdot)$ is Cobb-Douglas. The fixed labor cost, $\phi$, limits product proliferation in the long run and ties it to population growth, as discussed in detail in Peretto and Connolly (2007). Finally, the productivity of the firm depends on the stock of firm-specific knowledge, $Z_{i}$, with elasticity $\theta \in(0,1)$. Importantly, this firm-specific productivity term is Hicks-neutral with respect to labor and the resource.

The stock of firm-specific knowledge increases according to

$$
\dot{Z}_{i}(t)=\alpha K(t) L_{Z_{i}}(t)
$$

where $L_{Z_{i}}$ is labor employed in $\mathrm{R} \& \mathrm{D}$, and $\alpha>0$ a constant parameter. The productivity of $\mathrm{R} \& \mathrm{D}$ effort is determined by the exogenous parameter $\alpha$ and by the stock of public knowledge, $K$. Public knowledge accumulates as a result of spillovers. When one firm generates a new idea, it also generates non-excludable knowledge that benefits the R\&D of other firms. Specifically, we assume that

$$
K(t)=\int_{0}^{N(t)} \frac{1}{N(t)} Z_{i}(t) d i
$$

As discussed in detail in Peretto and Smulders (2002), this is the simplest specification of the spillover function that eliminates the strong scale effect in models of this class. 
Consider now a firm that starts to produce in instant $t$. Its present discounted value of the net cash flow is

$$
V_{i}(t)=\int_{t}^{\infty} \Pi_{i}(v) e^{-\int_{t}^{v}\left[r\left(v^{\prime}\right)+\delta\right] d v^{\prime}} d v
$$

where $\Pi_{i}$ is the instantaneous profit, $r$ is the instantaneous interest rate and $\delta>0$ is the instantaneous death rate of firms. ${ }^{4}$ In each instant, the firm chooses the cost-minimizing combination of rival inputs, $L_{X_{i}}$ and $R_{i}$, and the output level $X_{i}$ that maximize static profits $\Pi_{i}$ subject to the demand schedule coming from the household's problem. Given this choice, the monopolist then determines the time path of R\&D employment $L_{Z_{i}}$ that maximizes present-value profits (9) subject to the R\&D technology (7), taking as given the other firms' innovation paths. The solution to this problem is described in detail in the Appendix and yields the maximized value of the firm given the time path of the mass of firms.

\subsection{Horizontal Innovation (Entry)}

Outside entrepreneurs hire labor to perform $R \& D$ that develops new products and then set up firms to serve the market. This process of horizontal innovation increases the mass of firms over time. We assume that for each entrant, denoted $i$ without loss of generality, the labor requirement translates into a sunk cost that is proportional to the value of production: denoting by $L_{N_{i}}$ the labor employed in start-up activity, the entry cost is $w L_{N_{i}}=\beta Y_{i}$, where $Y_{i} \equiv P_{i} X_{i}$ is the value of production of the new good when it enters the market and $\beta>0$ is a parameter representing technological opportunity. This assumption captures the notion that entry requires more effort the larger the anticipated volume of production. ${ }^{5}$ The value of the firm entering the market at time $t$ equals the maximized present-value net cash flow $V_{i}(t)$ because, once in the market, the firm solves an intertemporal problem identical to that of

\footnotetext{
${ }^{4}$ The main role of the instantaneous death rate is to avoid the asymmetric dynamics and associated hysteresis effects that arise when entry entails a sunk cost. Such unnecessary complications would distract attention from the main point of the paper.

${ }^{5}$ Our assumption on the entry cost can be rationalized in several ways and does not affect the generality of our results. Peretto and Connolly (2007), in particular, discuss alternative formulations of the entry cost that yield the same qualitative properties for the equilibrium dynamics of the mass of firms that we exploit here.
} 
the generic incumbent. Free entry, therefore, requires

$$
V_{i}(t)=\beta Y_{i}(t)=w(t) L_{N_{i}}(t)
$$

for each entrant.

\section{Equilibrium Conditions}

The intertemporal choices of households and the profit-maximizing behavior of firms characterize the equilibrium path of the economy. This section describes consumption and fertility decisions, the dynamics of innovation rates and the relevant marketclearing conditions.

\subsection{Consumption and Fertility Choices}

Consumption and fertility choices are the solutions to the household's problem (see the Appendix for derivations). Consumption expenditure obeys the standard Keynes-Ramsey rule

$$
\dot{Y}(t) / Y(t)=r(t)-\rho .
$$

To characterize fertility choice, we denote by $\ell(t)$ the dynamic multiplier associated to constraint (3). This is the marginal shadow value of bringing into the world a future worker. The fertility rate must then satisfy the condition

$$
\ell(t)=\frac{1}{Y(t)} \cdot \psi w(t)-\frac{\mu}{b(t) L(t)},
$$

which says that the marginal value of an additional family member equals the net marginal utility cost of having children at each point in time. ${ }^{6}$ The dynamics of $\ell(t)$ are governed by the costate equation

$$
\rho \ell(t)-\dot{\ell}(t)=\frac{\sigma}{L(t)}+\frac{w(t)(1-\psi b(t))}{Y(t)}+\ell(t) \cdot(b(t)-d)
$$

\footnotetext{
${ }^{6}$ The first term in the right hand side of (12) is the gross marginal cost of child rearing in terms of foregone wage income, $\psi w(t)$, expressed in utility terms (i.e., multiplied by the marginal utility from consumption expenditure, $1 / Y(t)$ ). The second term is the direct marginal utility from increased population. The right hand side of (12) thus equals the net marginal cost of having children.
} 
which has the usual asset-pricing interpretation: each new born is an asset that delivers dividends in the future, directly as an adult family member and indirectly as an adult wage earner.

\subsection{Innovation Rates}

The solution to the monopolist problem yields a symmetric equilibrium across firms (see Appendix). We can thus write aggregate quantities of labor employed in production and vertical $\mathrm{R} \& \mathrm{D}$ as $L_{X}=N L_{X_{i}}$ and $L_{Z}=N L_{Z_{i}}$, respectively. Similarly, total resource use in manufacturing production equals $R=N R_{i}$. The firm's knowledge stock is $Z_{i}=Z$ for each $i \in[0, N]$ and evolves according to

$$
\frac{\dot{Z}(t)}{Z(t)}=\alpha \frac{L_{Z}(t)}{N(t)} .
$$

Equation (14) implies that an equilibrium in which employment per firm in vertical $\mathrm{R} \& \mathrm{D}$ is constant is characterized by a constant growth rate of firms' productivity. Symmetry across firms also yields that the value of a firm's production is $Y_{i}(t)=$ $Y(t) / N(t)$ so that the free-entry condition (10) is identically satisfied as $V_{i}=$ $\beta Y(t) / N(t)$ by every entrant. As a consequence, denoting total employment in start-up operations by $L_{N}=(\dot{N}+\delta N) \cdot L_{N_{i}}$, the net increase in the mass of firms equals

$$
\frac{\dot{N}(t)}{N(t)}=\frac{w(t)}{\beta Y(t)} \cdot L_{N}(t)-\delta
$$

The rates of vertical and horizontal innovation in (14) and (15) are interdependent

through the no-arbitrage condition that the associated returns must be equal (see Appendix).

\subsection{Market Clearing Conditions}

The economy's resource endowment is denoted by $\Omega$ and is constant over time. We assign full and well-defined property rights over this endowment to the household and, for simplicity, assume full utilization of the resource stock. In equilibrium, therefore, we have

$$
R(t)=\Omega,
$$


where $R(t)=N(t) R_{i}(t)$ is total resource use in production. Accordingly, the household's resource income equals $p(t) \Omega$ at each point in time. Labor market clearing requires that total labor supply be exhausted by its competing uses:

$$
L(t)(1-\psi b(t))=L_{X}(t)+L_{Z}(t)+L_{N}(t) .
$$

In the market for assets, equilibrium requires that the value of the household's portfolio equal the value of the securities issued by firms: $A(t)=N(t) V_{i}(t)=$ $\beta Y(t)$. Substituting this result into the wealth constraint (4), and using the saving rule (11), we obtain

$$
Y(t)=\frac{1}{1-\beta \rho}[w(t) L(t)(1-\psi b(t))+p(t) \Omega] .
$$

Expression (18) says that the ratio of household consumption expenditure, $Y(t)$, to household labor and resource income, the term in square brackets, is constant over time.

\section{General Equilibrium Dynamics}

For clarity, we split the analysis of dynamics in two parts. First, we study the interplay of population and resource scarcity (section 4.1). Second, we describe the interaction between horizontal and vertical innovations in determining productivity growth (section 4.2). A crucial characteristic of the resulting dynamics is the existence of a steady state displaying constant population associated to constant growth of (real) consumption per capita. Henceforth, we take labor as the numeraire and set $w(t) \equiv 1$

\subsection{Fertility and Resource Scarcity}

In this subsection we characterize the interactions between population and resource scarcity as a dynamic system involving two variables: the resource endowment per capita and the shadow value of humanity. The resource endowment per capita, $\omega(t) \equiv \Omega / L(t)$, is a state variable that is given at time zero but is subsequently driven by fertility choices via the dynamics of population. The shadow value of humanity is denoted by $h(t) \equiv \ell(t) L(t)$, where $\ell(t)$ is the marginal shadow value 
of a new worker previously defined, and is a forward-looking variable driving fertility choice under perfect foresight.

We derive the dynamical system in two steps. In section 4.1.1, we treat the values of $\omega(t)$ and $h(t)$ as given at time $t$ and derive the equilibrium values of the fertility rate, the resource price and consumption expenditure per capita. Building on this result, in section 4.1.2 we derive the two-by-two system that describes the joint dynamics of $\omega(t)$ and $h(t)$.

\subsubsection{Fertility, Expenditure and Resource Price}

The definition of $h(t)$ and the optimality condition (12) yield

$$
b(t)=\frac{\mu}{\frac{\psi}{y(t)}-h(t)} .
$$

Expression (19) shows that the fertility rate is positively related to consumption expenditure per capita, $y(t) \equiv Y(t) / L(t)$, given the shadow value of humanity. Consumption expenditure per capita, in turn, satisfies the equilibrium condition (18), which can be rewritten as

$$
y(t)=\frac{1-\psi b(t)+p(t) \omega(t)}{1-\beta \rho} .
$$

Equation (20) says that consumption expenditure per capita is proportional to the sum of labor income per capita, $1-\psi b(t)$, and resource income per capita, $p(t) \omega(t)$. Resource income per capita, in turn, is determined by the equilibrium between the demand for the resource by firms and the household's supply. Firms' conditional demand for the resource is (see Appendix)

$$
p(t) \omega(t)=y(t) \frac{\epsilon-1}{\epsilon} S(p(t)),
$$

where $S(p) \in(0,1)$ is the cost share of resource use, i.e., the ratio between total resource rents paid by firms to resource owners and the total variable costs of manufacturing production, and is a function of the resource price. Expression (21) specifies how expenditure decisions determine resource income through the endogenous resource price. This relationship depends on the characteristics of the production technology (6). Specifically, the cost share of resource use is increasing 
or decreasing in the resource price depending on the elasticity of input substitution (see Appendix):

$$
\frac{\partial S(p)}{\partial p}\left\{\begin{aligned}
>0 & \text { if }\left(L_{X_{i}}, R_{i}\right) \text { are complements; } \\
<0 & \text { if }\left(L_{X_{i}}, R_{i}\right) \text { are substitutes; } \\
=0 & \text { if } F(\cdot, \cdot) \text { is Cobb-Douglas. }
\end{aligned}\right.
$$

The cost-share effect summarized in (22) plays a crucial role in our results.

To see this, note that equations (19), (20) and (21) form a static system in three unknowns that determines the equilibrium levels of $p(t), y(t)$ and $b(t)$ for given levels of the resource endowment, $\omega(t)$, and the shadow value of humanity, $h(t)$. Figure 1 describes graphically the equilibrium determination (see the Appendix for details). In the upper panel, the loci obtained from (20) and (21) determine expenditure given fertility, $\bar{y}(b(t) ; \omega(t))$. In the lower panel, $\bar{y}(b(t) ; \omega(t))$ is combined with the locus (19) to determine the equilibrium expenditure and fertility, $y^{*}(\omega(t), h(t))$ and $b^{*}(\omega(t), h(t))$. The upper graphs of Figure 1 show that equilibrium expenditure responds differently to the resource endowment per capita, $\omega(t)$, depending on whether labor and resources are complements or substitutes. ${ }^{7}$ We summarize the relevant comparative-statics effects in the following Proposition.

Proposition 1 Given $(\omega(t), h(t))=(\omega, h)$ at instant $t$, there exists a unique triple

$$
\left\{p^{*}(\omega, h), y^{*}(\omega, h), b^{*}(\omega, h)\right\}
$$

determining the equilibrium levels of the fertility rate, consumption expenditure per capita and the resource price. Holding $h$ fixed, the marginal effects of an increase in $\omega$ are:

(i) Complementarity: $\quad \partial p^{*}(\omega, h) / \partial \omega<0, \quad \partial y^{*}(\omega, h) / \partial \omega<0, \quad \partial b^{*}(\omega, h) / \partial \omega<0$;

(ii) Substitutability: $\quad \partial p^{*}(\omega, h) / \partial \omega<0, \quad \partial y^{*}(\omega, h) / \partial \omega>0, \quad \partial b^{*}(\omega, h) / \partial \omega>0$;

(iii) Cobb-Douglas: $\quad \partial p^{*}(\omega, h) / \partial \omega<0, \quad \partial y^{*}(\omega, h) / \partial \omega=0, \quad \partial b^{*}(\omega, h) / \partial \omega=0$.

\footnotetext{
${ }^{7}$ In graphical terms, an increase in $\omega$ implies that the locus $y_{2}$ - which represents equation (20) - rotates counter-clock-wise whereas the locus $y_{3}$ - which represents equation (21) - is unaffected. Consequently, an increase in $\omega$ induces a decline in $\bar{y}(b ; \omega)$ under complementarity (diagram (a)), an increase in $\bar{y}(b ; \omega)$ under substitutability (diagram $(b)$ ), and no effect on $\bar{y}(b ; \omega)$ in the CobbDouglas case (diagram (c)).
} 
Holding $\omega$ fixed, the marginal effects of an increase in $h$ are

$$
\partial p^{*}(\omega, h) / \partial h<0, \quad \partial y^{*}(\omega, h) / \partial h<0, \quad \partial b^{*}(\omega, h) / \partial h>0,
$$

independently of the elasticity of input substitution.

Proposition 1 establishes four results. First, the effect of an increase in the resource endowment per capita $\omega$ on the equilibrium resource price $p^{*}$ is always negative. Second, the effect of $\omega$ on equilibrium consumption expenditure per capita $y^{*}$ is negative (positive) if labor and resources are complements (substitutes). The reason is that the sign of the effect of an increase in $\omega$ on resource income per capita depends on labor-resource substitutability. Under complementarity, resource demand is relatively inelastic and an increase in resource supply generates a drastic - that is, more than one-for-one - reduction of the price. Consequently, resource income, $p \omega$, falls and drives down consumption expenditure. Under substitutability, resource demand is relatively elastic and the increase in $\omega$ generates a mild reduction in the resource price, which implies a positive net effect on resource income and thereby higher consumption expenditure. In the special Cobb-Douglas case, the price and quantity effects exactly compensate each other so that resource income and expenditure are not affected by scarcity: $\partial(p \omega)^{*} / \partial \omega=0$ and $\partial y^{*} / \partial \omega=0$.

The third result in Proposition 1 is that $b^{*}(\omega, h)$ reacts to $\omega$ in the same direction as consumption expenditure: an increase in $\omega$ reduces (increases) the fertility rate if labor and resources are complements (substitutes). The reason is the optimality condition (19), which says that fertility rises with expenditure per capita for a given shadow value of humanity. The fourth result is that the marginal effects of an increase in $h$ do not depend on input substitutability. On the one hand, the fertility rate is higher the higher is the shadow value of humanity. On the other hand, consumption expenditure and the resource price decline with $h$ (for given $\omega$ ) because a higher fertility rate implies reduced work time.

These results play a key role in determining the equilibrium path of the economy: the qualitative characteristics of the transitional dynamics change depending on how income reacts to increased resource scarcity. We address this point by exploiting the instantaneous equilibrium defined in Proposition 1 to determine the joint dynamics of $\omega(t)$ and $h(t)$. 


\subsubsection{Dynamic System}

From (3), the differential equation describing the equilibrium dynamics of $\omega(t)$ is

$$
\dot{\omega}(t)=\omega(t) \cdot\left[d-b^{*}(\omega(t), h(t))\right]
$$

Using $h(t) \equiv \ell(t) L(t)$, the costate equation (13) becomes

$$
\dot{h}(t)=\rho h(t)-\sigma-\frac{1-\psi b^{*}(\omega(t), h(t))}{y^{*}(\omega(t), h(t))} .
$$

The system formed by (23) and (24) allows us to analyze the general equilibrium dynamics of the resource-population ratio and the associated shadow value of humanity. The results in Proposition 1 then allow us to characterize how expenditure per capita, the fertility rate and the resource price evolve along this path. Before studying in detail the properties of system (23)-(24), we complete the description of the general equilibrium dynamics by considering innovation rates and productivity growth.

\subsection{Innovations and Productivity Growth}

With the wage rate normalized to unity, the model's relevant measure of real output is the consumption term in the utility function (2). In equilibrium, therefore, the growth rate of the economy, $G(t)$, is (see Appendix)

$$
G(t)=\frac{\dot{y}(t)}{y(t)}-S(p(t)) \cdot \frac{\dot{p}(t)}{p(t)}+\left\{\theta \cdot \frac{\dot{Z}(t)}{Z(t)}+\frac{1}{\epsilon-1} \cdot \frac{\dot{N}(t)}{N(t)}\right\},
$$

where the last term in curly brackets represents the growth rate of total factor productivity (TFP), determined by vertical and horizontal innovations.

Since R\&D activity entails a fixed, sunk cost, developing vertical and horizontal innovations is profitable only if the firm's volume of production is large enough: there thus exist thresholds of market size, $Y(t)$, below which vertical innovation or horizontal innovation, or both, are inactive because firms cannot obtain a rate of return equal to the prevailing interest rate in the economy. ${ }^{8}$ These thresholds

\footnotetext{
${ }^{8}$ As shown in the Appendix (see the proof of Lemma 2), these thresholds are associated to critical levels of firm size and determine regions of the phase space where vertical and/or horizontal innovations shut down.
} 
play an important role in the characterization of early development phases - where "no-innovation traps" plausibly arise - but have no crucial bearing on the present analysis, which focuses on the future behavior of an economy that has already transited to "modern" production. Consequently, we henceforth assume that the values of the relevant parameters and the initial conditions $(L(0), N(0))$ are such that both vertical and horizontal innovations are active - see the Appendix for details.

The following Lemma establishes that, in equilibrium, the rates of vertical and horizontal innovation are jointly determined by two variables: firm size, denoted by $x(t) \equiv Y(t) / N(t)$, and the interest rate.

Lemma 2 Along the equilibrium path, the rates of vertical and horizontal innovation are, respectively:

$$
\begin{gathered}
\frac{\dot{Z}(t)}{Z(t)}=x(t) \frac{\alpha \theta(\epsilon-1)}{\epsilon}-r(t)-\delta ; \\
\frac{\dot{N}(t)}{N(t)}=\frac{1}{\beta}\left[\frac{1-\theta(\epsilon-1)}{\epsilon}-\frac{1}{x(t)}\left(\phi-\frac{r(t)+\delta}{\alpha}\right)\right]-\rho-\delta .
\end{gathered}
$$

The behavior of firm size, $x(t)$, is governed by the differential equation

$$
\dot{x}(t)=\frac{\alpha \phi-r(t)-\delta}{\alpha \beta}-\frac{1-\theta(\epsilon-1)-\beta \epsilon(r(t)+\delta)}{\beta \epsilon} \cdot x(t) .
$$

From (28), the evolution of firm size depends on the equilibrium path of the interest rate. The interest rate, in turn, follows the dynamics of aggregate market size, that reflect households' consumption and fertility choices: from (3) and (11), we have

$$
r(t)=\rho+\frac{\dot{y}(t)}{y(t)}+b(t)-d=\rho+\frac{\dot{Y}(t)}{Y(t)} .
$$

These results highlight the functioning of the "modern economy" as captured by the model structure. The interest rate carries all the information that firms need in order to choose paths of vertical and horizontal R\&D that are consistent with the evolution of the market for manufacturing goods. The path of aggregate market size depends on the evolution of the economy's resource base, that is, on the path of population.

Before analyzing population dynamics, we characterize the behavior of productivity growth when the economy converges to a steady state where nominal expenditure per capita, population and the resource price are constant. Equations (25) 
and (29) imply that in such a steady state, the interest rate equals $r(t)=\rho$, and the economy's real growth rate equals the TFP growth rate. Then, the following result holds:

Proposition 3 Suppose that the long-run equilibrium of the economy exhibits $\lim _{t \rightarrow \infty} \dot{y}(t)=$ $\lim _{t \rightarrow \infty} \dot{p}(t)=\lim _{t \rightarrow \infty} \dot{L}(t)=0$. Then, the net rate of horizontal innovation is zero and income growth is exclusively driven by vertical innovation,

$$
\lim _{t \rightarrow \infty} \frac{\dot{N}(t)}{N(t)}=0 \quad \text { and } \quad \lim _{t \rightarrow \infty} G(t)=\theta \cdot \lim _{t \rightarrow \infty} \frac{\dot{Z}(t)}{Z(t)} .
$$

Provided that parameters satisfy $\rho+\delta<\frac{\alpha \phi \theta(\epsilon-1)}{1-\beta \epsilon(\rho+\delta)}$, the growth rate is strictly positive:

$$
\lim _{t \rightarrow \infty} G(t)=\theta \frac{\theta(\epsilon-1)[\alpha \phi-(\rho+\delta)]}{1-\theta(\epsilon-1)-\beta \epsilon(\rho+\delta)}-\theta(\rho+\delta)>0
$$

Proposition 3 incorporates two main results concerning equilibria with constant population. First, as discussed in detail in Peretto (1998) and Peretto and Connolly (2007), economic growth in the long run is exclusively driven by vertical innovation: the process of entry enlarges the mass of goods until the gross entry rate matches the firms' death rate. Consequently, the mass of firms is constant and each firm invests a constant amount of labor in vertical $R \& D$. The second result is that, in the long run, real income growth is independent of factor endowments because net entry eliminates the strong scale effect. This property allows the economy to exhibit equilibria in which population is constant but real income per capita grows at a constant, endogenous rate. The next section addresses this point in detail.

\section{Population, Resources and Technology}

This section characterizes the equilibrium path of population and derives the main results of this paper. Population dynamics determine the supply of labor and the extent of resource scarcity at each point in time. An important property of the model is that the path of population can be studied in isolation from market size and innovation rates: system (23)-(24) fully captures the interactions between fertility and resource scarcity, and generates the equilibrium paths of population and resource use underlying the dynamics of aggregate market size. Then, as explained in section 
4.2, aggregate market size and the interest rate induced by population dynamics determine the evolution of firm size and, ultimately, total factor productivity growth.

Subsection 5.1 introduces the notion of pseudo-Malthusian steady state. The stability properties of the steady state crucially depend on the elasticity of substitution between labor and the natural resource in manufacturing production: section 5.2 discusses strict complementarity and strict susbstitutability, whereas section 5.3 considers the special Cobb-Douglas case. Subsection 5.4 considers a more general environment in which the input elasticity of substitution varies with the resource price and the economy may transit from a region of complementarity to a region of substitutability and viceversa.

\subsection{The Pseudo-Malthusian Steady State}

Consider a steady state $\left(\omega^{s s}, h^{s s}\right)$ in which both the resource per capita and the shadow value of humanity are constant. Imposing $\dot{\omega}=\dot{h}=0$ in the dynamic system (23)-(24), we obtain

$$
\begin{aligned}
d & =b^{*}\left(\omega^{s s}, h^{s s}\right), \\
h^{s s} & =\frac{1}{\rho} \cdot\left(\sigma+\frac{1-\psi d}{y^{*}\left(\omega^{s s}, h^{s s}\right)}\right) .
\end{aligned}
$$

Equation (32) is the obvious requirement of zero net fertility for constant population. Equation (33) defines the stationary shadow value of humanity. From Proposition 1 , the steady-state equilibrium $\left(\omega^{s s}, h^{s s}\right)$ also implies stationary values for the resource price, consumption expenditure and the fertility rate, which we denote by $\left(p^{s s}, y^{s s}, b^{s s}\right)$. In particular, we have (see Appendix):

Proposition 4 Assume $\psi \rho>1-\psi d$. Then, there exists a steady state where expenditure per capita and population are, respectively:

$$
\begin{aligned}
y^{s s} & =\frac{\psi \rho-(1-\psi d)}{\sigma+\mu(\rho / d)} \\
L^{s s} & =\frac{p^{s s}}{y^{s s}(1-\beta \rho)-(1-\psi d)} \cdot \Omega,
\end{aligned}
$$

Recall that by Proposition 3, given the constant values $\left(p^{s s}, y^{s s}, b^{s s}\right)$, real income growth equals the constant rate of vertical innovation. An important characteristic 
of this steady state is that $y^{s s}$ and $L^{s s}$ are independent of technology. From (34), expenditure per capita depends solely on preferences and demographic parameters: neither the endowment of the natural resource, $\Omega$, nor total factor productivity play any role. From (35), population is proportional to the resource endowment but remains independent of technology, while real income per capita grows at the endogenous rate (31). Therefore, we have a pseudo-Malthusian steady state, that is, a steady state with the Malthusian property that resource scarcity limits the population level, but where real income grows at an endogenous rate driven by technological change. ${ }^{9}$ Before pursuing this property further, we need to assess whether and under what circumstances the pseudo-Malthusian steady state is the long-run equilibrium achieved by the economy.

\subsection{Stability in the Cases of Global Substitutability and Global Complementarity}

The stability properties of the pseudo-Malthusian steady state depend on the input elasticity of substitution in manufacturing. We thus have three main cases: complementarity, substitutability and unit elasticity (Cobb-Douglas). In this subsection we concentrate on strict complementarity and strict substitutability.

Considering the $(\omega, h)$ plane, denote by $h^{(\dot{\omega}=0)}$ the stationary locus of resource per capita obtained from $(23)$ and by $h^{(\dot{h}=0)}$ the stationary locus for the shadow value of humanity obtained from (24). The phase diagrams for the cases of strict substitutability and strict complementarity, reported in Figure 2, yield the following result:

\footnotetext{
${ }^{9}$ It should be clear that a key assumption driving this result is that the technological change that drives long-run growth - i.e., vertical innovations - is Hicks-neutral with respect to labor and land. It is possible to introduce land-augmenting technological change, as in UGT, but doing so would complicate the model without adding insight to this paper's research question. In UGT models, land-augmenting technological change lifts the economy out of the Malthusian trap by allowing population growth in the phase where the subsistence consumption constraint is binding. In the present paper, instead, we focus on the future prospects for economic growth and therefore abstract from the characterization of early stages of development. Consequently, we do not need to postulate a bias of technological change that puts downward pressure on the land to population ratio.
} 
Proposition 5 Under substitutability, the stationary loci $h^{(\dot{h}=0)}$ and $h^{(\dot{\omega}=0)}$ are decreasing, $h^{(\dot{h}=0)}$ cuts $h^{(\dot{\omega}=0)}$ from below, and $\left(\omega^{s s}, h^{s s}\right)$ is saddle-point stable. Consequently, the pseudo-Malthusian steady state is the global attractor of the system and represents the long-run equilibrium of the economy. Under complementarity, the stationary loci are increasing, $h^{(\dot{h}=0)}$ cuts $h^{(\dot{\omega}=0)}$ from above, and $\left(\omega^{s s}, h^{s s}\right)$ is an unstable node. Consequently, the pseudo-Malthusian steady state is a separating threshold: if the resource is initially scarce (abundant) relative to labor, the economy experiences demographic explosion (collapse) in the long run.

Proposition 5 establishes that the pseudo-Malthusian steady state is the longrun equilibrium of the economy if labor and the resource are substitutes in production. Under complementarity, instead, the steady state is unstable and the economy follows diverging equilibrium paths leading to population explosion or collapse depending on the relative abundance of resources at time zero. ${ }^{10}$

The economic intuition for these results follows from the income effect of resource scarcity established in Proposition 1. First, consider the case of substitutability in diagram (a) and suppose that the resource is initially abundant, that is, $\omega_{0}>$ $\omega^{s s}$. During the transition, population grows and $\omega$ declines while the resource price, $p$, rises. Crucially, when labor and resources are substitutes, the price effect due to increasing land scarcity is not very strong and the economy experiences falling resource income per capita, pw, and, consequently, a falling fertility rate (cf. Proposition 1). Symmetrically, if the resource is relatively scarce at time zero, $\omega_{0}<$ $\omega^{s s}$, population shrinks during the transition and $\omega$ rises while $p$ falls. Since the price effect is weak, resource income per capita pw rises, driving the fertility rate up. In both cases, the transition ends when the fertility rate equals the death rate. Hence, under substitutability the pseudo-Malthusian steady state is the global attractor of the system because population growth generates resource income dynamics that yield self-balancing feedback effects: as land scarcity tightens, the price of land rises,

\footnotetext{
${ }^{10}$ For brevity we focus on the case in which the steady-state loci intersect and the pseudoMalthusian steady state exists. It should be clear, however, that the global dynamics are well defined even when the loci do not intersect. In such cases the equilibrium path is unique: the economy converges either to the attractor featuring constant exponential population growth (point E) or to the attractor featuring population collapse (this is the divergent path that approaches asymptotically the $\dot{h}=0$ locus as $\omega \rightarrow \infty$ ).
} 
but less than one for one with the endowment, so that land income per capita falls.

Now consider the case of complementarity in Figure 2, diagram (b). In this scenario, the pseudo-Malthusian steady state is not the global attractor of the system because the resource income effect is reversed. If the resource is initially scarce, $\omega_{0}<\omega^{s s}$, the dynamics exacerbate scarcity because, as population growth reduces $\omega$, the resource price $p$ rises more than one for one yielding a rise of resource income per capita $p \omega$ and a rise in fertility (cf. Proposition 1). This implies a feedback effect whereby population grows faster and drives the economy further away from the pseudo-Malthusian steady state. Resource per capita $\omega$ then tends asymptotically to zero as the economy experiences a demographic explosion. Symmetrically, if the resource is relatively abundant at time zero, $\omega_{0}>\omega^{s s}$, population shrinks and the increase in $\omega$ reduces resource income per capita via strong reductions in the resource price $p$, yielding a negative effect on fertility. Hence, under complementarity, the pseudo-Malthusian steady state is not the global attractor of the system because population growth generates resource income dynamics that yield self-reinforcing feedback effects on fertility choice.

The mechanism generating extinction under complementarity is quite different from that suggested by bio-economic models in which collapse is due to overexploitation of the natural resource base - see, e.g., D'Alessandro (2007) and, especially, Taylor (2009). Conversely, the demographic collapse in our model is due to an excessive scarcity of manpower that prevents the economy from taking advantage of the natural resource base. This situation is self-reinforcing because the low resource income per capita yields below-replacement fertility and further population decline. Moreover, as we highlighted in the discussion of the dynamics of the innovation rates, the collapse of the population eventually results in the shutting down of $R \& D$ activity and ultimately of modern manufacturing production itself.

Our results are novel also with respect to UGT because the qualitative dynamics described in Figure 2 are generated by a price effect that does not arise if there is no resource market - as in Galor and Weil (2000) - or, if there is, when labor and resources exhibit a unit elasticity of substitution, as in Lucas (2002). To make this point transparent, we now turn to the Cobb-Douglas case and show that the pseudo-Malthusian equilibrium is indeed created by the resource price effect. 


\subsection{The Special Cobb-Douglas Case}

When the manufacturing technology takes the Cobb-Douglas form, the pseudoMalthusian steady state does not exist and the model predicts that population grows, or shrinks, forever at a constant rate. The proof follows from Proposition 1. A unit elasticity of input substitution implies that neither expenditures nor the fertility rate are affected by variations in resources per capita. Consequently, the stationary loci $h^{(\dot{\omega}=0)}$ and $h^{(\dot{h}=0)}$ become horizontal straight lines. The properties of the dynamic system (23)-(24) fall in three subcases: (i) the locus $h^{(\dot{\omega}=0)}$ lies below $h^{(\dot{h}=0)}$, (ii) the locus $h^{(\dot{\omega}=0)}$ lies above $h^{(\dot{h}=0)}$, or (iii) the two loci coincide.

Figure 3 describes the phase diagram (see the Appendix for details) in all subcases. The common characteristic is that, given the initial condition $\omega(0)=\omega_{0}$, the shadow value of humanity jumps on the $h^{(\hat{h}=0)}$ locus at time zero. ${ }^{11}$ In subcase (i) there is no pseudo-Malthusian equilibrium: given $\omega_{0}$, the economy moves along the $h^{(\dot{h}=0)}$ locus and population grows at a constant exponential rate during the whole transition. $^{12}$ Subcase (ii) is specular: the economy moves along the $h^{(\dot{h}=0)}$ locus with a permanently declining level of population, with no transitional dynamics in the fertility rate because the shadow value of humanity is constant. In subcase (iii), the parameters are such that the equilibrium fertility rate exactly coincides with the exogenous mortality rate. However, this steady state is different from the pseudoMalthusian equilibrium as there are no interactions between resource scarcity and fertility over time: the economy maintains the initial resource endowment per capita $\omega_{0}$ forever.

\subsection{A More General Characterization}

In this subsection we generalize our characterization of global dynamics by postulating that the cost share function $S(p)$ changes the sign of its derivative at some

\footnotetext{
${ }^{11}$ All explosive paths yielding $h(t) \rightarrow \infty$ or $h(t)<0$ at some finite date are ruled out by standard arguments: they either violate the transversality condition $\lim _{t \rightarrow \infty} h(t) e^{-\rho t}=0$ or the household's budget constraint.

${ }^{12}$ The population growth rate is constant because the shadow value of humanity is constant. Since expenditure is not affected by variations in resource per capita (cf. Proposition 1), having $\dot{h}=0$ implies a constant fertility rate $b(t)$ by virtue of condition (19). The same reasoning applies to all subcases.
} 
price $\bar{p}$ corresponding to a level of the land/population ratio $\bar{\omega}$. We consider the following two cases.

Case I. Assume $S^{\prime}(p)<0$ for $p<\bar{p}$ (i.e., for $\omega>\bar{\omega}$ ); $S^{\prime}(p)=0$ for $p=\bar{p}$ (i.e., for $\omega=\bar{\omega}$ ); and $S^{\prime}(p)>0$ for $p>\bar{p}$ (i.e., for $\omega<\bar{\omega}$ ). In this scenario, the technology has the property that it is easier to substitute land for labor when land is used relatively abundantly. That is, when land is relatively cheap, the demand for land is elastic. As population grows and $\omega$ falls, the relative price of land rises and, at some point, it crosses the threshold $\bar{p}$ entering the region where land demand becomes inelastic, signaling that land becomes complementary to labor. This property produces the phase diagram in Figure 5, graph (a), which combines our previous results on substitutability and complementarity: the steady-state loci are increasing and concave for low values of $\omega$, and then decreasing and convex for high values of $\omega$. The peaks of both loci correspond exactly to the value $\bar{\omega}$ associated to the critical price $\bar{p}$. Our previous analysis implies that, for the same set of restrictions on the parameters, there exists a pseudo-Malthusian steady state within each region - i.e., the two loci exhibit one intersection in the substitutability region and one intersection in the complementarity region. As $S^{\prime}(p)$ changes sign smoothly, the two interior steady states have opposite stability properties: the one in the substitutability region (the one to the right in Figure 5, graph (a)) is saddle-path stable; the one in the complementarity region (the one to the left) is unstable. The global dynamics of the economy are then straightforward. The state space in split in two basins of attraction: for initial values of $\omega$ larger than the complementarity steady state, the economy jumps on the saddle path that converges to the substitutability steady state; for values of $\omega$ smaller than the complementarity steady state, the economy jumps on the saddle path that converges to point $E$, that is, the path of exponential population explosion.

Case II. $S^{\prime}(p)>0$ for $p>\bar{p}$ (i.e., for $\omega<\bar{\omega}$ ); $S^{\prime}(p)=0$ for $p=\bar{p}$ (i.e., for $\omega=\bar{\omega}$ ); and $S^{\prime}(p)>0$ for $p<\bar{p}$ (i.e., for $\omega>\bar{\omega}$ ). This scenario postulates that substitutability holds for high land price whereas complementarity holds for low land price. We obtain a different combination of the two baseline phase diagrams, in which the sequence of regions is inverted with respect to Case I. The stationary loci are decreasing and convex for low $\omega$, and increasing and concave for high $\omega$. 
The phase diagram in Figure 5, graph (b), displays two intersections with opposite stability properties: the one in the substitutability region is saddle-path stable; the one in the complementarity region is unstable. The characteristics of global dynamics are evident: for initial values of $\omega$ smaller than the complementarity steady state, the economy jumps on the saddle path that converges to the substitutability steady state; for values of $\omega$ larger than the complementarity steady state, the economy jumps on the saddle path that converges to the path of extinction (note that, in Case II, point $E$ has lost stability).

The above analysis hinges on the hypothesis that the cost share of land is a nonmonotonic function of the land price. Since the conventional production functions - included the translog function, which is one of the most flexible - yield cost share functions that are monotonic in $p$, we construct an example by assuming that the technology of the production sector determines a unit cost function that deviates from the Cobb-Douglas case according to the value of a parameter. Suppose the unit cost function is

$$
C(p)=p^{S_{C D}} \cdot \frac{p+k}{p+1}
$$

where $p^{S_{C D}}$ is the cost share of land in the Cobb-Douglas case, and $k>0$ determines the deviation from the Cobb-Douglas case. This technology satisfies all the properties of standard neoclassical production functions. The associated cost share function is ${ }^{13}$

$$
S(p)=\frac{d \log C(p)}{d \log p}=S_{C D}+\frac{p(1-k)}{(p+1)(p+k)} .
$$

When $0<k<1$, the cost share is always larger than $S_{C D}$ and is hump-shaped in $p$ with a maximum at $\bar{p}=\sqrt{k}$. In contrast, when $k>1$, the cost share is always smaller than $S_{C D}$ and is U-shaped in $p$ with a minimum at $\bar{p}=\sqrt{k}$.

An alternative way of generating a non-monotonic cost share functions is to extend our model to a vertical production structure, along the lines set forth in Peretto (2012). ${ }^{14}$

\footnotetext{
${ }^{13}$ To ensure that $S(p)>0$ for all $p$, we impose the technical restriction that $S(\bar{p})>0$.

${ }^{14}$ With vertical production structures, the economy may endogenously transit from a region of complementarity to a region of substitutability (or vice versa). For example, see Peretto (2012) and Peretto and Valente (2012), which both have production structures with two vertically related sec-
} 


\section{Minimum Resource Requirement: A Dominant Pre- ventive Check}

A question that arises naturally is whether the prediction of stable population in the long run - which holds under substitutability - extends to the cases of complementarity and unit substitution if we further enrich the model. In particular, considering the equilibrium paths yielding $\omega(t) \rightarrow 0$, what kind of forces may stop the demographic explosion? In this section we present a simple extension of the model that yields a considerable generalization of our results. Specifically, we assume that each adult has a fixed minimum requirement of the resource and show that this induces a congestion effect that operates through the price of land $p$ and amplifies the preventive check against demographic explosion making it the dominant force for any elasticity of substitution between labor and land.

\subsection{Equilibrium with Minimum Requirement}

We denote the fixed requirement of resource per adult by $\eta$, and impose

$$
\omega(t) \geqslant \eta \text { in each } t \in[0, \infty) .
$$

This assumption can be rationalized in many ways. For example, if the resource $\Omega$ represents total available land, constraint (36) establishes that there is a minimum land requirement - e.g., residential land - for each adult. Setting $\eta=0$, we are back to the original model analyzed in the previous sections.

When $\eta>0$, the minimum requirement restricts the supply of the resource to firms: the dynamic wealth constraint (4) is replaced by

$$
\dot{A}(t)=r(t) A(t)+w(t) L(t)(1-\psi b(t))+p(t) \cdot(\Omega-\eta L(t))-Y(t),
$$

where $R(t)=\Omega-\eta L(t)$ is aggregate net resource supply. The rest of the economy is as before. The representative household maximizes welfare (1) subject to (37)

tors - resource-intensive primary production upstream and manufacturing production downstream

- that trough a combination of complementarity upstream and substitutability downstream produce a pattern for the whole economy that exhibits an overall cost share of land that is initially decreasing and then increasing in the land price. 
and conditions (20) and (21) are replaced by

$$
\begin{aligned}
& y(t)=\frac{1-\psi b(t)+p(t) \cdot(\omega(t)-\eta)}{1-\beta \rho}, \\
& y(t)=\frac{p(t) \cdot(\omega(t)-\eta)}{\frac{\epsilon-1}{\epsilon} S(p(t))},
\end{aligned}
$$

respectively. Using (38), (39) and the static fertility relation (19) - which is unchanged - we can follow the same steps taken in section 4.1 .1 and build a static system that determines the conditional equilibrium levels of expenditure, the resource price and the fertility rate.

The only modification is that the relevant index of resource supply per capita is not $\omega(t)$ but the net resource supply per capita, which we denote by $s(t) \equiv \omega(t)-\eta$. We thus obtain the same results described in Proposition 1: treating $s(t)$ and $h(t)$ as given at time $t$, there exists a unique triple

$$
\left\{p^{*}(s(t), h(t)), \quad y^{*}(s(t), h(t)), \quad b^{*}(s(t), h(t))\right\}
$$

determining the equilibrium levels of $p(t), y(t)$ and $b(t)$ and displaying the same comparative-statics properties listed in Proposition 1. Moreover (see Appendix),

$$
\lim _{s(t) \rightarrow 0^{+}} p^{*}(s(t), h(t))=\infty
$$

which says that the resource price goes to infinity when the resource endowment per capita approaches the minimum threshold.

\subsection{Steady State with Minimum Requirement}

The dynamics of $s(t)$ are governed by

$$
\dot{s}(t)=(s(t)+\eta) \cdot\left[d-b^{*}(s(t), h(t))\right] .
$$

The differential equation for $h(t),(24)$, is now replaced by (see Appendix)

$$
\dot{h}(t)=\rho h(t)-\sigma-\frac{1-\psi b^{*}(s(t), h(t))-\eta \cdot p^{*}(s(t), h(t))}{y^{*}(s(t), h(t))} .
$$

In steady state expenditure per capita is (see Appendix)

$$
y^{*}\left(s^{s s}, h^{s s}\right)=\frac{\psi \rho-(1-\psi d)\left[1+\left(\eta / s^{s s}\right)\right]}{\sigma+\mu(\rho / d)-\left(\eta / s^{s s}\right)(1-\beta \rho)},
$$


where $s^{s s}$ indicates the constant level of net resource supply per capita associated to the constant level of the shadow value of humanity $h^{s s}$.

Denote by $h^{(\dot{s}=0)}$ and $h^{(\dot{h}=0)}$ the stationary loci determined by (41) and (42) in the $(s, h)$ plane. The difference with respect to the original model with $\eta=0$ is that the locus $h^{(\dot{h}=0)}$ exhibits a vertical asymptote in $h=0$ and goes to minus infinity independently of the elasticity of substitution. This result is described in the upper panel of Figure 4, where we consider (a) substitutability, (b) complementarity and (c) the Cobb-Douglas case for the relevant subcase featuring growing population. ${ }^{15}$ Starting from $\eta=0$, and subsequently imposing higher values of $\eta$, the left branch of the locus $h^{(\dot{h}=0)}$ bends downward and the locus shifts down. Crucially, the congestion effect due to the minimum land requirement per capita removes $\omega=0$ as a possible attractor of the system: for technologies displaying a substitution elasticity below or equal to one, we obtain a new steady state featuring constant population.

The intuition is provided by result (40) combined with the costate equation (42). When the amount of resource per capita approaches the minimum requirement, the resource price explodes to infinity. This force affects fertility choices through the term $\eta \cdot p(s, h)$ in equation (42) that governs the dynamics of the shadow value of humanity: differently from the baseline model with $\eta=0$, the representative household now reduces fertility in response to the exploding resource price which signals the excessive congestion of the resource.

\subsection{Dynamics with Minimum Requirement}

Figure 4 clarifies the consequences of the minimum resource requirement for the number of steady states and their stability properties. Under substitutability, our conclusions do not change: as shown in diagram (d), the steady state $\left(s^{s s}, h^{s s}\right)$ is unique and is saddle-point stable because $h^{(\dot{h}=0)} \operatorname{cuts} h^{(\dot{s}=0)}$ from below.

Under complementarity, instead, two steady states arise. As shown in diagram (e), there is a "low" pseudo-Malthusian equilibrium, denoted by $\left(s_{s s}^{\prime}, h_{s s}^{\prime}\right)$, as well as a "high" pseudo-Malthusian equilibrium, denoted by $\left(s_{s s}^{\prime \prime}, h_{s s}^{\prime \prime}\right)$. The "high" equi-

\footnotetext{
${ }^{15}$ In the Cobb-Douglas scenario, we limit our attention to subcase (i) because it is the only case featuring demographic explosion (cf. Figure 3). The introduction of a minimum resource requirement is only relevant for equilibrium paths that exhibit zero resource per capita in the long run.
} 
librium is an unstable node and thus acts as a separating threshold. The "low" equilibrium, instead, is saddle-path stable and is therefore the local attractor of the system. Provided that the initial net resource endowment is below $s_{s s}^{\prime \prime}$, the economy converges to the pseudo-Malthusian steady state $\left(s_{s s}^{\prime}, h_{s s}^{\prime}\right)$.

Consider now the Cobb-Douglas case. In the original model with $\eta=0$ the economy follows a path of constant exponential growth of population (cf. Figure 3, subcase (i)). The positive requirement $\eta>0$ generates a unique pseudo-Malthusian steady state that is saddle-path stable and is thus the global attractor of the system; see Figure 4, diagram (f). We summarize these conclusions as follows.

Proposition 6 Given a fixed requirement of resource per adult $\eta>0$, the economy converges towards a pseudo-Malthusian steady state under substitutability, under complementarity (provided that $s_{0}<s_{s s}^{\prime \prime}$ ) and under Cobb-Douglas technology (subcase (i)).

With the inclusion of a congestion effect due to a minimum resource requirement that mimics the role of residential land, our theory of the long-run level of population appears robust with respect to scenarios in which labor and resources are not strictly substitutes: the preventive-check mechanism driven by the price of land always eliminates explosive population dynamics.

\section{Conclusion}

This paper investigated the links between resource scarcity, income levels and population growth in a Schumpeterian model with endogenous fertility. Our analysis offers the following results. When labor and resources are strict complements or strict substitutes in production, the increase in resource scarcity induced by population growth generates price effects that modify income per capita yielding feedback effects on fertility. These price effects create a pseudo-Malthusian equilibrium in which population is constant, income per capita grows at a constant endogenous rate, and population size is independent of technology. Under substitutability, this equilibrium is a global attractor and indeed determines the population level in the long run: increased resource scarcity reduces income producing self-balancing effects on population via reduced fertility. Under complementarity, instead, the pseudo-Malthusian 
equilibrium is a separating threshold and the population level follows diverging paths: increased (reduced) resource scarcity generated by the growth (decline) of population increases (decreases) income per capita and fertility rates, implying selfreinforcing feedback effects that drive the economy towards demographic explosion (human extinction).

In a more general environment where the input elasticity of substitution varies with the resource price, the economy may transit from a region of complementarity to a region of substitutability and viceversa: the long-run equilibrium becomes state dependent, because there are two steady states with distinct basins of attraction, and the economy may converge towards the pseudo-Malthusian steady state or diverge from it depending on the initial level of the resource endowment.

Further results arise by introducing a minimum resource requirement per adult e.g., residential land. In this case, the economy avoids demographic explosion in all scenarios because agents internalize the minimum requirement in their intertemporal choices, enhancing the preventive check that generates stable pseudo-Malthusian equilibria. Accordingly, population achieves a stationary level in the long run even under complementarity.

Our analysis unveils a theory of interactions between resources and population that differs in several respects from the existing literature. Price effects are generally neglected in Unified Growth Theory, which typically assumes no resource markets or a unitary elasticity of substitution between labor and resources. Bio-economic models, on the other hand, often allow for price effects driven by scarcity, but if we consider the extinction path arising under complementarity in our model, the collapse of the society is not due to excessive natural scarcity - as in models where natural regeneration becomes negative below a certain stock threshold - but rather to the continuous decline in resource prices which reduces expenditures, incomes and fertility. In other words, the problem is not scarcity of natural resources but of the labor needed to exploit those resources. More generally, our analysis suggests a theory of the population level which is consistent with the fact that Planet Earth has a finite carrying capacity of people. This basic characteristic of closed systems is not captured by standard balanced growth models, that typically predict exponential population growth in the long run. 


\section{References}

Barro, R., Sala-i-Martin, X. (2004). Economic Growth - Second Edition. Cambridge MA: MIT Press.

Basener, B., Ross, D. (2005). Booming and crashing populations and Easter island. SIAM Journal of Applied Mathematics 65 (2): 684-701.

Birdsall, N., Sinding, S.W. (2001). How and Why Population Matters: New Findings, New Issues. In Birdsall, N., Kelley, A.C. ans Sinding, S.W. (eds.), Population matters: Demographic change, economic growth, and poverty in the developing world, pp. 3-23. Oxford and New York: Oxford University Press.

Bloom, D., Canning, D. (2001). Cumulative Causality, Economic Growth, and the Demographic Transition. In Birdsall, N., Kelley, A.C. ans Sinding, S.W. (eds.), Population matters: Demographic change, economic growth, and poverty in the developing world, pp. 165-198. Oxford and New York: Oxford University Press.

Brander, J., Taylor, M. (1998). The simple economics of Easter island: A Ricardo Malthus model of renewable resource use. American Economic Review 88 (1): 119-138.

Connolly, M., Peretto, P.F. (2003). Industry and the Family: Two Engines of Growth. Journal of Economic Growth 8: 115-145.

D'Alessandro, S. (2007). Non-linear dynamics of population and natural resources: The emergence of different patterns of development. Ecological Economics 62 (3-4): 473-481.

Dalton, T., Coase, R., Badiollah, R. (2005). Renewable resources, property-rights regimes and endogenous growth. Ecological Economics, 52, 31-41.

Dinopoulos, E., Thompson, P. (1998). Schumpeterian growth without scale effects. Journal of Economic Growth 3 (4): 313-335. 
Galor, O. (2005). From stagnation to growth: unified growth theory. In P. Aghion and S.N. Durlauf (eds) Handbook of Economic Growth, Vol. 1A, pp. 171-293. Elsevier: Amsterdam.

Galor, O. (2011). Unified Growth Theory. Princeton NJ: Princeton University Press.

Galor, O., Weil, D.N. (2000). Population, technology and growth: From the Malthusian regime to the demographic transition. American Economic Review 110: 806-828.

Good, D.H., Reuveny, R. (2009). On the collapse of historical civilizations. American Journal of Agricultural Economics 91(4): 863-879.

Ha, J., Howitt, P. (2007). Accounting for Trends in Productivity and R\&D: A Schumpeterian Critique of Semi-Endogenous Growth Theory. Journal of Money, Credit, and Banking 33: 733-74.

Harford, J. (2000). Methods of pricing common property use and some implications for optimal child-bearing and the social discount rate. Resource and Energy Economics 22: 103-124.

Kelley, A. (2001). The Population Debate in Historical Perspective: Revisionism Revised. In Birdsall, N., Kelley, A.C. ans Sinding, S.W. (eds.), Population matters: Demographic change, economic growth, and poverty in the developing world, pp. 24-54. Oxford and New York: Oxford University Press.

Kremer, M. (1993). Population Growth and Technological Change: One Million B.C. to 1990. Quarterly Journal of Economics 108: 681-716.

Laincz, C., Peretto, P.F. (2006). Scale Effects in Endogenous Growth Theory: An Error of Aggregation, not Specification. Journal of Economic Growth 11:263288.

Lucas, R. (2002). Lectures on Economic Growth. Cambridge MA: Harvard University Press. 
Madsen, J.B. (2008). Semi-endogenous versus Schumpeterian Growth Models: Testing the Knowledge Production Function Using International Data. Journal of Economic Growth 13: 1-26.

Madsen, J.B., Ang, J.B. (2011). Can Second-Generation Endogenous Growth Models Explain the Productivity Trends in the Asian Miracle Economies? Review of Economics and Statistics, forthcoming.

Madsen, J.B., Ang, J.B., Banerjee, R. (2010). Four centuries of British economic growth: the roles of technology and population. Journal of Economic Growth 15: 263-290.

Malthus, T.R. (1798). An Essay on the Principle of Population. St. Paul's ChurchYard, London.

Nerlove, M., Raut, L.K. (1997). Growth Models with Endogenous Population: A General Framework. In M. R. Rosenzweig and O. Stark (eds.), Handbook of Population and Family Economics, Vol. 1B. Amsterdam: Elsevier Science, North-Holland.

Peretto, P.F. (1998). Technological Change and Population Growth. Journal of Economic Growth 3 (4): 283-311.

Peretto, P.F. (2012). Resource abundance, growth and welfare: A Schumpeterian perspective. Journal of Development Economics 97: 142-155.

Peretto, P.F., Connolly, M. (2007). The Manhattan Metaphor. Journal of Economic Growth 12 (4): 329-350.

Peretto, P.F., Smulders, S. (2002). Technological Distance, Growth And Scale Effects. Economic Journal 112 (481): 603-624.

Reuveny, R., Decker, C. (2000). Easter island: Historical anecdote or warning for the future? Ecological Economics 35: 271-287.

Robinson, J., Srinivasian, T.N. (1997). Long-term consequences of population growth: Technological change, natural resources, and the environment. In 
M. R. Rosenzweig and O. Stark (eds.), Handbook of Population and Family Economics, Vol. 1B. Amsterdam: Elsevier Science, North-Holland.

Taylor, M.S. (2009). Innis Lecture: Environmental crises: past, present, and future. Canadian Journal of Economics 42 (4): 1240-1275.

Wang, P., Yip, C.K., Scotese, C.A. (1994). Fertility Choice and Economic Growth: Theory and Evidence. The Review of Economics and Statistics 76(2): 255-266. 

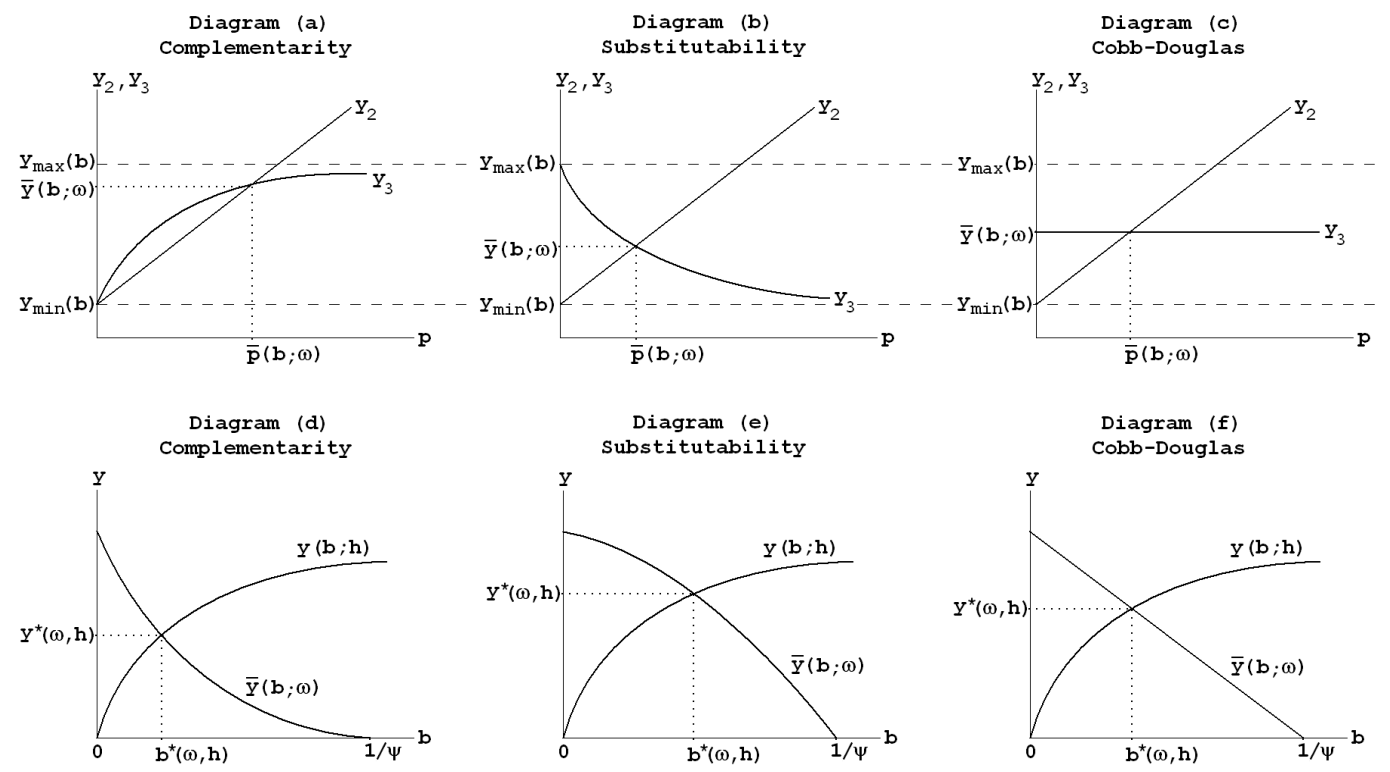

Figure 1: Equilibrium determination in system (19)-(20)-(21). In the upper graphs, the loci $y_{2}$ and $y_{3}$ represent equations (20) and (21), respectively, and the equilibrium determines $\bar{y}(b ; \omega)$. In the lower graphs, $\bar{y}(b ; \omega)$ is combined with the locus $y(b ; h)$, representing equation (19), to determine $y^{*}(\omega, h)$ and $b^{*}(\omega, h)$. See the proof of Proposition 1 for details. 
(a) Substitutes

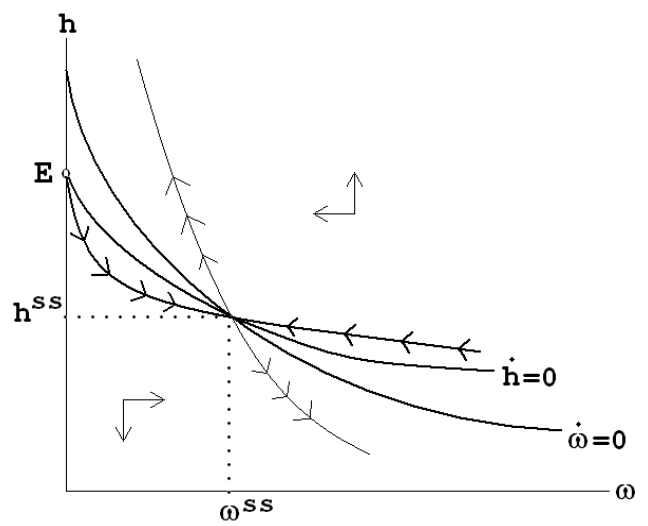

(b) Complements

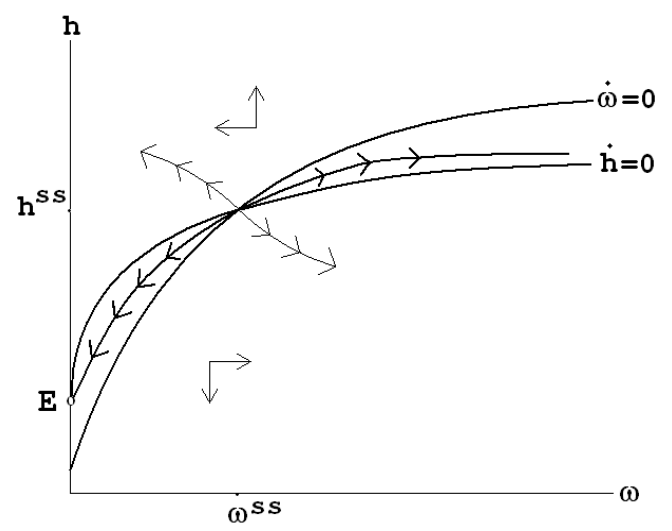

Figure 2: Phase diagrams of system (23)-(24) under substitutability (a) and complementarity (b). 

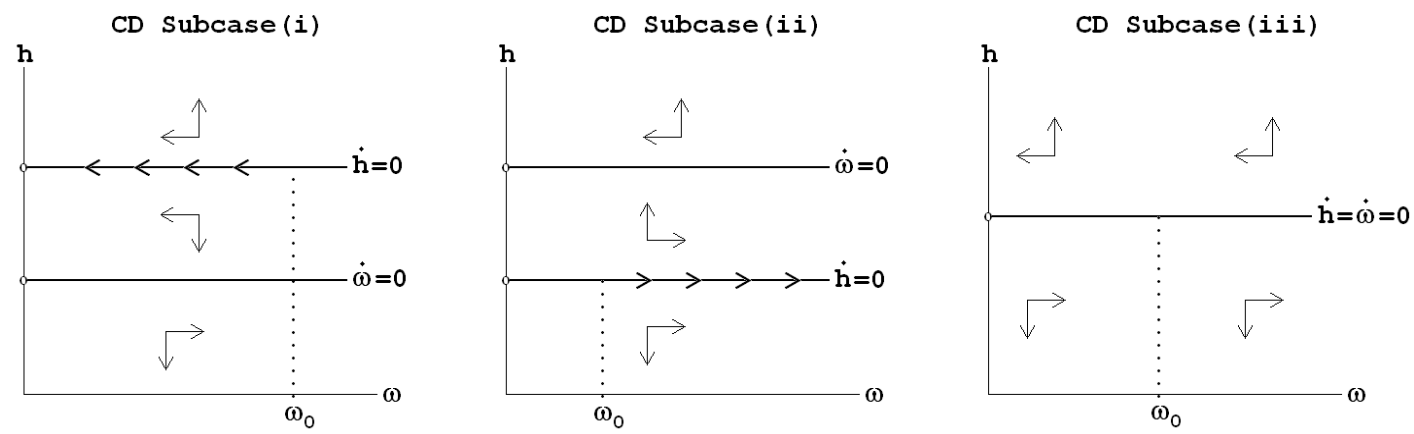

Figure 3: Phase diagrams of system (23)-(24) in the Cobb-Douglas case for three different parametrizations. 
(a) Substitutes

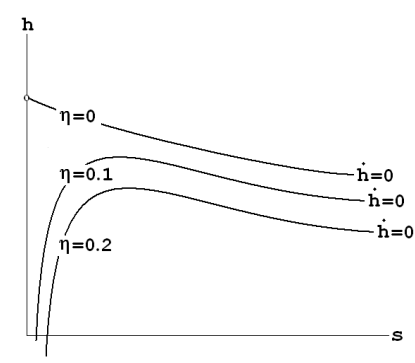

(d) Substitutes

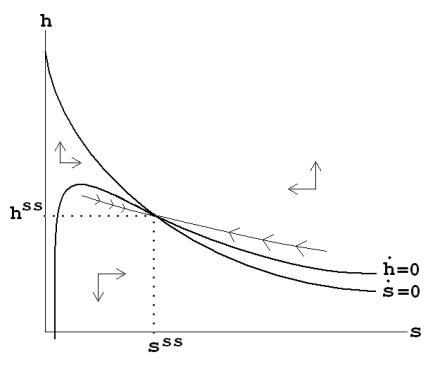

(b) Complements

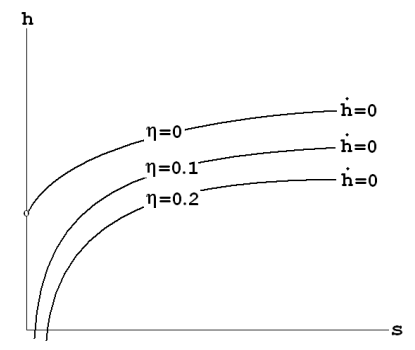

(e) Complements

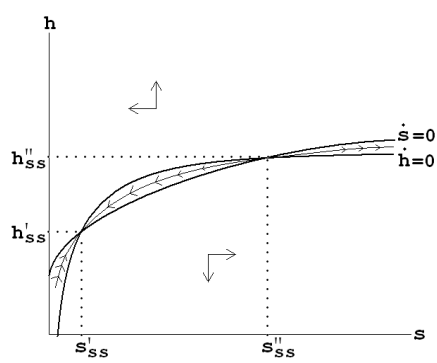

(c) Cobb-Douglas

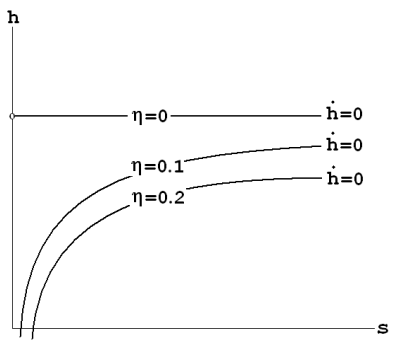

(f) Cobb-Douglas

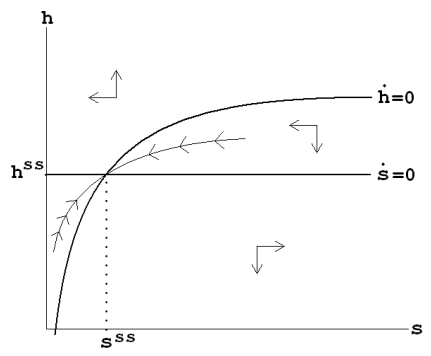

Figure 4: Dynamics in the model with minimum resource requirement. Upper panel: the stationary locus $h^{(\dot{h}=0)}$ obtained from (42) under substitutability (a), complementarity (b) and Cobb-Douglas (c) for increasing values of $\eta$. Lower panel: phase diagrams of system (41)-(42). 

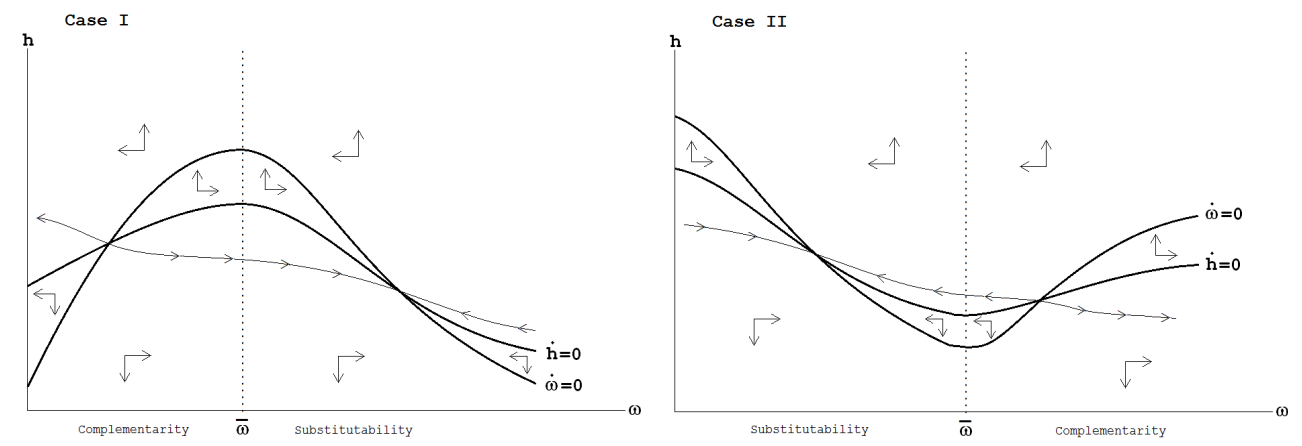

Figure 5: A more general characterization: two cases of reversal of the elasticity of substitution. 


\section{A Appendix For Reviewers}

Household problem: derivation of (11), (12), (13). In the first step, the household maximizes (2) subject to (5). The solution yields the demand schedule for product $i$,

$$
X_{i}(t)=Y(t) \frac{P_{i}(t)^{-\epsilon}}{\int_{0}^{N(t)} P_{i}(t)^{1-\epsilon} d i} .
$$

Atomistic firms take the denominator of (A.1) as given and each monopolist faces an isoelastic demand curve. Plugging (A.1) in (A.1), indirect instantaneous utility reads

$$
\log u(t)=\tilde{u}+\log (Y(t) / L(t))+\mu \log b(t)+(\sigma+1) \log L(t)
$$

where $\tilde{u}$ is a function of the goods' prices (taken as given). Expression (A.2) implies a positive net elasticity of utility to population, $\sigma>0$. In the second step, the household maximizes (1) subject to (4) and (3) using $Y$ and $b$ as control variables and $A$ and $L$ as state variables. Plugging (A.2) in (1), the Hamiltonian for this problem reads

$\mathcal{L}^{H} \equiv \tilde{u}+\log Y+\mu \log b+\sigma \log L+\lambda \cdot[r A+w L(1-\psi b)+p R-Y]+\ell \cdot[L(b-d)]$

where $\lambda$ and $\ell$ are the dynamic multipliers associated to $A$ and $L$, respectively. The optimality conditions read

$$
\begin{aligned}
1 / Y(t) & =\lambda(t) \\
\mu / b(t) & =\psi \lambda(t) w(t) L(t)-\ell(t) L(t), \\
\rho \lambda(t)-\dot{\lambda}(t) & =\lambda(t) r(t), \\
\rho \ell(t)-\dot{\ell}(t) & =(\sigma / L(t))+\lambda(t) w(t)(1-\psi b(t))+\ell(t) \cdot(b(t)-d),
\end{aligned}
$$

in addition to the usual transversality conditions

$$
\lim _{t \rightarrow \infty} \lambda(t) A(t) e^{-\rho t}=\lim _{t \rightarrow \infty} \ell(t) L(t) e^{-\rho t}=0 .
$$

Combining (A.4) with (A.6), we obtain (11). Substituting (A.4) in (A.5) and solving for $\ell(t)$, we obtain (12). Using (A.4) to eliminate $\lambda(t)$ from (A.7), we obtain equation (13) in the text. 
The monopolist problem. The instantaneous profit of the $i$-th monopolist is

$$
\Pi_{i}=P_{i} X_{i}-w L_{X_{i}}-p R_{i}-w L_{Z_{i}} .
$$

From (6), the cost-minimizing combination of $L_{X_{i}}$ and $R_{i}$, for given wage $w$ and resource price $p$, yields total production costs

$$
w \phi+C(w, p) Z_{i}^{-\theta} X_{i}
$$

where $C(\cdot)$ is the unit-cost function homogeneous of degree one. Using (A.10), instantaneous profits (A.9) can be re-written as

$$
\Pi_{i}=\left[P_{i}-C(w, p) Z_{i}^{-\theta}\right] X_{i}-w \phi-w L_{Z_{i}}
$$

where $P_{i}$ is the market price of the produced variety. In the first step, the monopolist maximizes (A.11) subject to the demand schedule (A.1) - taking the denominator as given - obtaining the mark-up rule

$$
P_{i}=\frac{\epsilon}{\epsilon-1} \cdot C(w, p) Z_{i}^{-\theta} \text { for each } i \in[0, N] .
$$

In the second step, the firm maximizes (9) subject to (7). Using (A.11), the currentvalue Hamiltonian for the $i$-th firm reads

$$
\mathcal{L}_{i}^{M} \equiv\left[P_{i}-C(w, p) Z_{i}^{-\theta}\right] X_{i}-w \phi-w L_{Z_{i}}+\zeta_{i} \alpha K L_{Z_{i}}
$$

where $\zeta_{i}$ is the dynamic multiplier attached to firm-specific knowledge. The state variable is $Z_{i}$, the control variable is $\mathrm{R} \& \mathrm{D}$ employment, $L_{Z_{i}}$, and the public knowledge stock, $K$, is taken as given. The first order conditions for the interior solution are given by

$$
\begin{aligned}
\zeta_{i} \alpha K & =w \\
r+\delta-\theta C(w, p) Z_{i}^{-\theta-1} \frac{X_{i}}{\zeta_{i}} & =\frac{\dot{\zeta}_{i}}{\zeta_{i}} \\
\lim _{v \rightarrow \infty} e^{-\int_{t}^{v}\left[r\left(v^{\prime}\right)+\delta\right] d v^{\prime}} \zeta_{i}(v) Z_{i}(v) & =0 .
\end{aligned}
$$

Peretto (1998, Proposition 1) shows that, under the restriction $1>\theta(\epsilon-1)$, the firm is always at the interior solution, where $w=\zeta_{i} \alpha K$ holds and equilibrium is 
symmetric. $^{16}$

Innovation rates and no-arbitrage. Symmetry across manufacturing firms implies $K=Z=Z_{i}$ and thereby $\dot{K} / K=\alpha L_{Z} / N$, where $L_{Z}$ is aggregate employment in vertical $\mathrm{R} \& \mathrm{D}$. Also, the free-entry condition (10) reduces to $V_{i}=$ $\beta Y(t) / N(t)$ by every $i$-th entrant in the manufacturing business. Time-differentiating (9), we have

$$
r(t)+\delta=\frac{\Pi_{X i}(t)}{V_{i}(t)}+\frac{\dot{V}_{i}(t)}{V_{i}(t)}
$$

which is a perfect-foresight, no-arbitrage condition for the equilibrium in the capital market. The rates of return to vertical and horizontal $R \& D$ read

$$
\begin{aligned}
& r(t)=r_{Z}(t) \equiv \frac{\dot{w}(t)}{w(t)}+\alpha\left[\frac{\theta(\epsilon-1)}{\epsilon} \cdot \frac{Y(t)}{N(t) w(t)}-\frac{L_{Z}(t)}{N(t)}\right]-\delta \\
& r(t)=r_{N}(t) \equiv \frac{1}{\beta}\left[\frac{1}{\epsilon}-\frac{N(t) w(t)}{Y(t)}\left(\phi+\frac{L_{Z}(t)}{N(t)}\right)\right]+\frac{\dot{Y}(t)}{Y(t)}-\frac{\dot{N}(t)}{N(t)}-(\delta A)
\end{aligned}
$$

respectively. Equation (A.17), is obtained by time-differentiating (A.13) and using the demand curve (A.1), the R\&D technology (7) and the price strategy (A.12). Equation (A.18) follows from substituting $V_{i}=\beta Y / N$ in (A.16). No-arbitrage requires that $r_{Z}(t)=r_{N}(t)$ in each instant $t$.

Conditional input demands: derivation of (21) and (22). The cost function (A.10) gives rise to the conditional factor demands

$$
L_{X_{i}}=\frac{\partial C(w, p)}{\partial w} Z_{i}^{-\theta} X_{i}+\phi \text { and } R_{i}=\frac{\partial C(w, p)}{\partial p} Z_{i}^{-\theta} X_{i}
$$

for each firm $i$. Combining (A.19) with the price strategy (A.12), and aggregating across firms under symmetry, we obtain the aggregate demand schedules

$$
\begin{aligned}
L_{X}(t) & =Y(t) \frac{\epsilon-1}{\epsilon}[1-\tilde{S}(w(t), p(t))]+\phi N(t), \\
R(t) & =Y(t) \frac{\epsilon-1}{\epsilon} \frac{\tilde{S}(w(t), p(t))}{p(t)},
\end{aligned}
$$

\footnotetext{
${ }^{16}$ Because the Hamiltonian is linear in $L_{Z_{i}}$, we may have three cases. If $\zeta_{i} \alpha K<w$, the value of the marginal unit of knowledge is below its cost and the firm does not invest. If $\zeta_{i} \alpha K>w$, the value of the marginal unit of knowledge exceeds its cost: this case is ruled out as it violates the general equilibrium conditions (the firm would demand an infinite amount of labor for $R \& D$ purposes).
} 
where $\tilde{S}(w, p)$ is the resource share in the firm's total variable cost:

$$
\tilde{S}(w, p) \equiv \frac{p R_{i}}{C(w, p) Z_{i}^{-\theta} X_{i}}=\frac{\partial \log C(w, p)}{\partial \log p} .
$$

Normalizing the wage rate $w(t)=1$ in each instant $t$, the cost function reduces to $C(w, p)=C(1, p)$ and the resource cost share can be re-defined as

$$
S(p) \equiv \tilde{S}(1, p)=\frac{\partial \log C(1, p)}{\partial \log p} .
$$

Because the resource cost share $S(p)$ equals the elasticity of the cost function with respect to the resource price, it exhibits the properties reported in (22) as well as

(i) Complementarity: $\quad \lim _{p \rightarrow 0} S(p)=0, \quad \lim _{p \rightarrow \infty} S(p)=1$,

(ii) Substitutability: $\quad \lim _{p \rightarrow 0} S(p)=1, \quad \lim _{p \rightarrow \infty} S(p)=0$,

(iii) Cobb-Douglas: $\quad S(p)=\bar{S} \in(0,1)$.

Multiplying both sides of (A.21) by $p(t) / L(t)$ and substituting $R(t)=\Omega$ from (16), we obtain equation (21) in the text.

Proof of Proposition 1. Eliminating time-arguments and treating $(\omega, h)$ as parameters, the system formed by (19), (20) and (21) can be rewritten as

$$
\begin{aligned}
y_{1}(b ; h) & \equiv \frac{\psi}{h+(\mu / b)}, \\
y_{2}(b, p ; \omega) & \equiv \frac{1-\psi b}{1-\beta \rho}+\frac{\omega}{1-\beta \rho} \cdot p, \\
y_{3}(b, p) & \equiv \frac{1-\psi b}{1-\beta \rho-\frac{\epsilon-1}{\epsilon} S(p)},
\end{aligned}
$$

where (A.27) is obtained by plugging (21) in (20) to eliminate $p \omega$ and solving for $y$. The proof of Proposition 1 involves three steps. First, we prove the existence and uniqueness of the equilibrium. Second, we assess the marginal effects of variations in $\omega$. Third, we assess the marginal effects of variations in $h$.

Step \#1. The subsystem formed by (A.26) and (A.27) can be represented graphically in the $(p, y)$ plane: given $b$ and $\omega$, function $y_{2}(b, p ; \omega)$ is a linear increasing function of $p$, whereas the behavior of $y_{3}(b, p)$ depends on the elasticity of substitution: from (22), it is (i) increasing and concave under complementarity; (ii) decreasing and convex under substitutability; (iii) a flat horizontal line in the CobbDouglas case. The three cases are respectively described in diagrams (a), (b) and 
(c) of Figure 1. The vertical intercepts and horizontal asymptotes of $y_{3}(b, p)$ are defined in (A.24) and (A.27). In all cases, the intersection $y_{2}(b, p ; \omega)=y_{3}(b, p)$ is unique and determines the conditional values $\bar{y}(b ; \omega)$ and $\bar{p}(b ; \omega)$. In particular, $\bar{y}(b ; \omega)$ exhibits the property

$$
y_{\min }(b) \equiv \frac{1-\psi b}{1-\beta \rho}<\bar{y}(b ; \omega)<\frac{1-\psi b}{1-\beta \rho-\frac{\epsilon-1}{\epsilon}} \equiv y_{\max }(b) .
$$

In all cases, a ceteris paribus increase in $b$ shifts down the vertical intercept of $y_{2}(b, p ; \omega)$ as well as the vertical intercept and the horizontal asymptotes of $y_{3}(b, p)$. This implies that $\bar{y}(b ; \omega)$ is monotonically decreasing in $b$ with

$$
\frac{\partial}{\partial b} \bar{y}(b ; \omega)<0, \quad \lim _{b \rightarrow 0} \bar{y}(b ; \omega)=\bar{y}(0 ; \omega)>0, \quad \lim _{b \rightarrow 1 / \psi} \bar{y}(b ; \omega)=0 .
$$

Reporting the $\bar{y}(b ; \omega)$ locus in the $(b, y)$ plane in the three cases, we respectively obtain diagrams (d), (e) and (f) in Figure 1. In the same diagrams, we plot the $y_{1}(b ; h)$ schedule defined in (A.25), which is strictly increasing and concave in $b$. In all cases, the intersection $\bar{y}(b ; \omega)=y_{1}(b ; h)$ is unique and thus determines a unique couple of equilibrium values, $b^{*}(\omega, h)$ and $y^{*}(\omega, h)$. Given $b^{*}(\omega, h)$ and $y^{*}(\omega, h)$, there exists a unique value of the resource price $p^{*}(\omega, h)$ satisfying the monotonous relation (A.26).

Step \#2. The marginal effects of $\omega$ can be studied by means of Figure 1. In all cases, an increase in $\omega$ increases the slope of $y_{2}(b, p ; \omega)$ leaving $y_{3}(b, p)$ unchanged, so that

$$
\frac{\partial \bar{p}(b ; \omega)}{\partial \omega}<0, \quad \lim _{\omega \rightarrow 0^{+}} \bar{p}(b ; \omega)=\infty, \quad \lim _{\omega \rightarrow \infty} \bar{p}(b ; \omega)=0,
$$

independently of the elasticity of substitution. With respect to $\bar{y}(b ; \omega)$, we have to consider each case in turn. Under complementarity, the positive effect of $\omega$ on the slope of $y_{2}(b, p ; \omega)$ implies

$$
\text { (Compl.): } \quad \frac{\partial \bar{y}(b ; \omega)}{\partial \omega}<0, \quad \lim _{\omega \rightarrow 0^{+}} \bar{y}(b ; \omega)=y_{\max }(b), \quad \lim _{\omega \rightarrow \infty} \bar{y}(b ; \omega)=y_{\min }(b)
$$

Result (A.31) implies that, in diagram (d) of Figure 1 , the $\bar{y}(b ; \omega)$ schedule moves down rotating counter-clockwise around the horizontal intercept $b=1 / \omega$. We thus have $\frac{\partial}{\partial \omega} y^{*}(\omega, h)<0$ and $\frac{\partial}{\partial \omega} b^{*}(\omega, h)<0$. From the equilibrium relation (A.27) evaluated in the equilibrium, the signs $\frac{\partial}{\partial \omega} y^{*}(\omega, h)<0$ and $\frac{\partial}{\partial \omega} b^{*}(\omega, h)<0$ require

$$
\frac{\partial S\left(p^{*}(\omega, h)\right)}{\partial \omega}=\frac{\partial S(p)}{\partial p} \cdot \frac{\partial p^{*}(\omega, h)}{\partial \omega}<0,
$$


which - given $\partial S(p) / \partial p>0$ - implies $\frac{\partial}{\partial \omega} p^{*}(\omega, h)<0$. Now consider the case of substitutability: the positive effect of $\omega$ on the slope of $y_{2}(b, p ; \omega)$ implies

$$
\text { (Subs.): } \quad \frac{\partial \bar{y}(b ; \omega)}{\partial \omega}>0, \quad \lim _{\omega \rightarrow 0^{+}} \bar{y}(b ; \omega)=y_{\min }(b), \quad \lim _{\omega \rightarrow \infty} \bar{y}(b ; \omega)=y_{\max }(b),
$$

Result (A.32) implies that, in diagram (e) of Figure 1, the $\bar{y}(b ; \omega)$ schedule moves up rotating clockwise around the horizontal intercept $b=1 / \omega$. We thus have $\frac{\partial}{\partial \omega} y^{*}(\omega, h)>0$ and $\frac{\partial}{\partial \omega} b^{*}(\omega, h)>0$. From the equilibrium relation (A.27) evaluated in the equilibrium, the signs $\frac{\partial}{\partial \omega} y^{*}(\omega, h)>0$ and $\frac{\partial}{\partial \omega} b^{*}(\omega, h)>0$ require

$$
\frac{\partial S\left(p^{*}(\omega, h)\right)}{\partial \omega}=\frac{\partial S(p)}{\partial p} \cdot \frac{\partial p^{*}(\omega, h)}{\partial \omega}>0
$$

which - given $\partial S(p) / \partial p<0$ - implies $\frac{\partial}{\partial \omega} p^{*}(\omega, h)<0$. Next consider the CobbDouglas case: the positive effect of $\omega$ on the slope of $y_{2}(b, p ; \omega)$ yields a decline in $\bar{p}(b ; \omega)$ but no effect on conditional expenditure, $\frac{\partial}{\partial \omega} \bar{y}(b ; \omega)=0$. This implies that, in diagram (f) of Figure 1, the $\bar{y}(b ; \omega)$ schedule is the horizontal line

$$
\text { (Cobb-Douglas): } \quad \bar{y}(b ; \omega)=\frac{1-\psi b}{1-\beta \rho-\frac{\epsilon-1}{\epsilon} \bar{S}},
$$

obtained by substituting the constant $S(p)=\bar{S} \in(0,1)$ in (A.27). Hence, we get $\frac{\partial}{\partial \omega} y^{*}(\omega, h)=0$ and $\frac{\partial}{\partial \omega} b^{*}(\omega, h)=0$. The only effect of $\omega$ is on the resource price, which is negative: $\frac{\partial}{\partial \omega} p^{*}(\omega, h)=\frac{\partial}{\partial \omega} \bar{p}(b ; \omega)<0$.

Step \#3. From definition (A.23), the combined function $\nu(p) \equiv p / S(p)$ exhibits

$$
\frac{\partial \nu(p)}{\partial p}>0, \quad \lim _{p \rightarrow 0} \nu(p)=0, \quad \lim _{p \rightarrow \infty} \nu(p)=\infty,
$$

and allows us to rewrite the equilibrium relation (21) as

$$
y^{*}(\omega, h)=\omega \cdot \frac{\epsilon}{\epsilon-1} \cdot \nu\left(p^{*}(\omega, h)\right) .
$$

The marginal effects of $h$ can be studied by means of diagrams (d), (e) and (f) in Figure 1. In all cases, an increase in $h$ leaves the $\bar{y}(b ; \omega)$ schedule unchanged whereas the $y_{1}(b ; h)$ schedule defined in (A.25) exhibits

$$
\frac{\partial y_{1}(b ; h)}{\partial h}<0, \quad \lim _{h \rightarrow 0^{+}} y_{1}(b ; h)=\frac{\psi}{\mu} b, \quad \lim _{h \rightarrow \infty} y_{1}(b ; h)=0 .
$$


Result (A.36) implies that $y_{1}(b ; h)$ moves down rotating clockwise around the origin as $h$ increases. Consequently, we have $\frac{\partial}{\partial h} b^{*}(\omega, h)>0$ and $\frac{\partial}{\partial h} y^{*}(\omega, h)<0$ independently of the elasticity of substitution. The effect of $h$ on the resource price is obtained by differentiating (A.35) with respect to $h$, which yields

$$
\frac{\partial}{\partial h} y^{*}(\omega, h)=\omega \frac{\epsilon}{\epsilon-1} \cdot \frac{\partial \nu}{\partial p} \cdot \frac{\partial p^{*}(\omega, h)}{\partial h} .
$$

Since $\partial \nu / \partial p>0$ independently of the elasticity of substitution, the sign of $\frac{\partial}{\partial h} p^{*}(\omega, h)$ is always the same as that of $\frac{\partial}{\partial h} y^{*}(\omega, h)<0$.

Derivation of (25). By symmetry in the manufacturing sector, substitute $X_{i}=X$ for each $i \in[0, N]$, the demand function (A.1), and the price strategy (A.12), in the consumption term of the utility function (2) to obtain

$$
\left[\int_{0}^{N(t)}\left(\frac{X_{i}(t)}{L(t)}\right)^{\frac{\epsilon-1}{\epsilon}} d i\right]^{\frac{\epsilon}{\epsilon-1}}=\frac{y(t)}{P_{i}(t)} N(t)^{\frac{1}{\epsilon-1}}=\frac{\epsilon-1}{\epsilon} \cdot \frac{y(t)}{C(1, p(t))} Z_{i}(t)^{\theta} N(t)^{\frac{1}{\epsilon-1}}
$$

where we have used $Z_{i}(t)=Z(t)$ and $C(w(t), p(t))=C(1, p(t))$. Recalling that $S(p) \equiv \frac{\partial \log C(1, p)}{\partial \log p}$ by definition (A.23), the growth rate of (A.37) is given by (25).

Proof of Lemma 2. Setting $w(t)=1$ and $Y(t) / N(t)$ in (A.17), and using (14) to eliminate $L_{Z} / N$, we obtain

$$
\frac{\dot{Z}(t)}{Z(t)}=\left\{\begin{array}{ll}
\frac{\alpha \theta(\epsilon-1)}{\epsilon} x(t)-r(t)-\delta & \text { if } x(t)>\tilde{x}_{Z}(t) \equiv \frac{\epsilon(r(t)+\delta)}{\alpha \theta(\epsilon-1)} \\
0 & \text { if } x(t) \leqslant \tilde{x}_{Z}(t)
\end{array},\right.
$$

where $x(t) \equiv Y(t) / N(t)$ is market size and $\tilde{x}_{Z}(t)$ is the threshold level of market size below which vertical innovation is inactive. This proves result (26). From (A.18), we have

$$
r(t)=\frac{1}{\beta}\left[\frac{1}{\epsilon}-\frac{1}{x(t)}\left(\phi+\frac{1}{\alpha} \cdot \frac{\dot{Z}(t)}{Z(t)}\right)\right]+\frac{\dot{Y}(t)}{Y(t)}-\frac{\dot{N}(t)}{N(t)}-\delta .
$$

Plugging $r(t)=\rho+\dot{Y}(t) / Y(t)$ from (11) and solving the resulting expression for $\dot{N}(t) / N(t)$, we obtain

$$
\frac{\dot{N}(t)}{N(t)}= \begin{cases}\frac{1}{\beta}\left[\frac{1-\theta(\epsilon-1)}{\epsilon}-\frac{1}{x(t)}\left(\phi-\frac{r(t)+\delta}{\alpha}\right)\right]-\rho-\delta & \text { if } x(t)>\tilde{x}_{N}(t) \equiv \frac{\phi-\frac{r(t)+\delta}{\alpha}}{\frac{1-\theta(\epsilon-1)}{\epsilon}-\beta(\rho+\delta)} \\ 0 & \text { if } x(t) \leqslant \tilde{x}_{N}(t)\end{cases}
$$


where $\tilde{x}_{N}(t)$ is the threshold level of market size below which horizontal innovation is inactive. This proves result (27). From (A.38), the growth rate of firm size equals

$$
\frac{\dot{x}(t)}{x(t)}=\frac{\dot{Y}(t)}{Y(t)}-\frac{\dot{N}(t)}{N(t)}=r(t)+\delta-\frac{1}{\beta}\left[\frac{1}{\epsilon}-\frac{1}{x(t)}\left(\phi+\frac{1}{\alpha} \cdot \frac{\dot{Z}(t)}{Z(t)}\right)\right],
$$

where we can substitute $(26)$ and solve for $\dot{x}(t)$ in the case $x(t)>\max \left\{\tilde{x}_{Z}(t), \tilde{x}_{N}(t)\right\}$ to obtain (28). To guarantee that firm size is sufficiently large throughout the transition, we assume that at time 0 agents select a pair $(\omega(0), h(0))$ such that

$$
x(0)=\frac{Y(0)}{N(0)}=\frac{L(0)}{N(0)} \cdot y(\omega(0), h(0))>\max \left\{\tilde{x}_{Z}(0), \tilde{x}_{N}(0)\right\},
$$

where

$$
\tilde{x}_{Z}(0)=\frac{\epsilon(r(0)+\delta)}{\alpha \theta(\epsilon-1)} \text { and } \tilde{x}_{N}(0)=\frac{\phi-\frac{r(0)+\delta}{\alpha}}{\frac{1-\theta(\epsilon-1)}{\epsilon}-\beta(\rho+\delta)} .
$$

The intuition for this assumption is that the condition $x(t)>\max \left\{\tilde{x}_{Z}(t), \tilde{x}_{N}(t)\right\}$ identifies a region in $(\omega, h)$ space where both $R \& D$ activities are turned on. The boundary of this region is a decreasing line that depends on parameters and the initial mass of firms $N(0)$, and such that the two R\&D activities are on for values $(\omega(t), h(t))$ below it. Consequently, given the model's parameters, we can always choose a combination of initial population, $L(0)$, resource endowment, $\Omega$, and initial mass of firms, $N(0)$, such that given $\omega(0)$ the economy selects a value $h(0)$ that implies $x(t)>\max \left\{\tilde{x}_{Z}(t), \tilde{x}_{N}(t)\right\}$ for all $t \geq 0$ on a path that converges to the steady state with constant population. Similarly, we can ensure that given $\omega(0)$ the economy selects a value $h(0)$ that implies $x(t)>\max \left\{\tilde{x}_{Z}(t), \tilde{x}_{N}(t)\right\}$ for some $t \geq 0$ on a path that diverges from the steady state with constant population.

Proof of Proposition 3. If $\lim _{t \rightarrow \infty} \dot{y}(t)=\lim _{t \rightarrow \infty} \dot{p}(t)=\lim _{t \rightarrow \infty} \dot{L}(t)=0$, equations (25) and (29) respectively imply

$$
\begin{aligned}
\lim _{t \rightarrow \infty} G(t) & =\theta \cdot \frac{\dot{Z}(t)}{Z(t)}+\frac{1}{\epsilon-1} \cdot \frac{\dot{N}(t)}{N(t)}, \\
\lim _{t \rightarrow \infty} r(t) & =\rho .
\end{aligned}
$$

Substituting (A.40) in $\tilde{x}_{Z}(t) \equiv \frac{\epsilon(r(t)+\delta)}{\alpha \theta(\epsilon-1)}$, the asymptotic critical threshold for obtaining positive vertical innovations is

$$
\lim _{t \rightarrow \infty} x(t)>\tilde{x}(\infty) \equiv \frac{\epsilon(\rho+\delta)}{\alpha \theta(\epsilon-1)} .
$$


Consequently, from (28), we have two possible steady-state levels for firm size,

$$
\lim _{t \rightarrow \infty} x(t)=\left\{\begin{array}{ll}
(\epsilon / \alpha) \cdot \frac{\alpha \phi-(\rho+\delta)}{1-\theta(\epsilon-1)-\beta \epsilon(\rho+\delta)} & \text { if } \rho+\delta<\frac{\alpha \phi \theta(\epsilon-1)}{1-\beta \epsilon(\rho+\delta)} \\
\frac{\epsilon \phi}{1-\beta \epsilon(\rho+\delta)} & \text { if } \rho+\delta \geqslant \frac{\alpha \phi \theta(\epsilon-1)}{1-\beta \epsilon(\rho+\delta)}
\end{array},\right.
$$

where $\rho+\delta<\frac{\alpha \phi \theta(\epsilon-1)}{1-\beta \epsilon(\rho+\delta)}$ implies strictly positive vertical innovations and $\rho+\delta \geqslant$ $\frac{\alpha \phi \theta(\epsilon-1)}{1-\beta \epsilon(\rho+\delta)}$ implies, instead, constant $Z(t)$ in the long run. Substituting (A.42) in (26), we have

$$
\lim _{t \rightarrow \infty} \frac{\dot{Z}(t)}{Z(t)}=\left\{\begin{array}{ll}
\frac{\theta(\epsilon-1)[\alpha \phi-(\rho+\delta)]}{1-\theta(\epsilon-1)-\beta \epsilon(\rho+\delta)}-(\rho+\delta) & \text { if } \rho+\delta<\frac{\alpha \phi \theta(\epsilon-1)}{1-\beta \epsilon(\rho+\delta)} \\
0 & \text { if } \rho+\delta \geqslant \frac{\alpha \phi \theta(\epsilon-1)}{1-\beta \epsilon(\rho+\delta)}
\end{array},\right.
$$

where the case of operative vertical $R \& D$ does not require any further restriction on parameters. Plugging (A.43) in (27), we also have

$$
\lim _{t \rightarrow \infty} \frac{\dot{N}(t)}{N(t)}=\left\{\begin{array}{ll}
0 & \text { if } \rho+\delta<\frac{\alpha \phi \theta(\epsilon-1)}{1-\beta \epsilon(\rho+\delta)} \\
0 & \text { if } \rho+\delta \geqslant \frac{\alpha \phi \theta(\epsilon-1)}{1-\beta \epsilon(\rho+\delta)}
\end{array} .\right.
$$

Using (A.39), results (A.43) and (A.44) imply (30) and (31).

Derivation of (34) and (35). Using (32) and (33) to eliminate $b^{s s}$ and $h^{s s}$ from the equilibrium conditions (19) and (20), respectively, we obtain expressions (34) and (35) in the text.

Proof of Proposition 5. Proposition 5 is proved in three steps. First, we show that the stationary loci $h^{(\dot{\omega}=0)}$ and $h^{(\dot{h}=0)}$ are both increasing (decreasing) in the $(\omega, h)$ plane under complementarity (substitutability), and are both horizontal straight lines in the special Cobb-Douglas case. Second, we show that under substitutability (complementarity), $h^{(\dot{h}=0)}$ cuts $h^{(\dot{\omega}=0)}$ from below (above). Third, we show that under substitutability (complementarity), the steady state $\left(\omega^{s s}, h^{s s}\right)$ is a stable saddle-point (unstable node or focus).

Step \#1. Equation (34) and condition (A.27) evaluated at $\left(y^{s s}, b^{s s}\right)$ imply

$$
\sigma+\mu(\rho / d)=\frac{\psi \rho-(1-\psi d)}{1-\psi d} \cdot\left[1-\beta \rho-\frac{\epsilon-1}{\epsilon} S(p)\right]
$$

For future reference, define the constants

$$
\Gamma_{0} \equiv 1-\beta \rho-\frac{\epsilon-1}{\epsilon} \text { and } \Gamma_{1} \equiv 1-\beta \rho,
$$


where $\Gamma_{0}<\Gamma_{1}$. Imposing $\dot{\omega}=0$ in $(23)$, the resulting locus $h^{(\dot{\omega}=0)}$ is characterized by $b^{*}(\omega, h)=d$. From the Proof of Proposition 1 , we know that, given $b^{*}(\omega, h)=d$, the equilibrium is characterized by the conditional values $\bar{y}(b ; \omega)$ and $\bar{p}(b ; \omega)$ determined in Figure 1 and evaluated in $b=d$. In particular, results (A.31), (A.32) and (A.33) respectively imply the three cases

$$
\left\{\begin{array}{llll}
\text { Comp: } & \frac{\partial \bar{y}(d ; \omega)}{\partial \omega}<0, & \lim _{\omega \rightarrow 0} \bar{y}(d ; \omega)=\frac{1-\psi d}{\Gamma_{0}}, & \lim _{\omega \rightarrow \infty} \bar{y}(d ; \omega)=\frac{1-\psi d}{\Gamma_{1}} \\
\text { Subs: } & \frac{\partial \bar{y}(b ; \omega)}{\partial \omega}>0, & \lim _{\omega \rightarrow 0} \bar{y}(d ; \omega)=\frac{1-\psi d}{\Gamma_{1}}, & \lim _{\omega \rightarrow \infty} \bar{y}(d ; \omega)=\frac{1-\psi d}{\Gamma_{0}} ; \\
\text { C-D: } & \frac{\partial \bar{y}(b ; \omega)}{\partial \omega}=0, & \bar{y}(d ; \omega)=\frac{1-\psi d}{1-\beta \rho-\frac{\epsilon-1}{\epsilon} \bar{S}} &
\end{array}\right\}
$$

From (19), given $b^{*}(\omega, h)=d$, the locus $h^{(\dot{\omega}=0)}$ is represented by

$$
h^{(\dot{\omega}=0)}=\frac{\psi}{y^{*}(\omega, h)}-\frac{\mu}{b^{*}(\omega, h)}=\frac{\psi}{\bar{y}(d ; \omega)}-\frac{\mu}{d} .
$$

Plugging results (A.47) in (A.48), we obtain

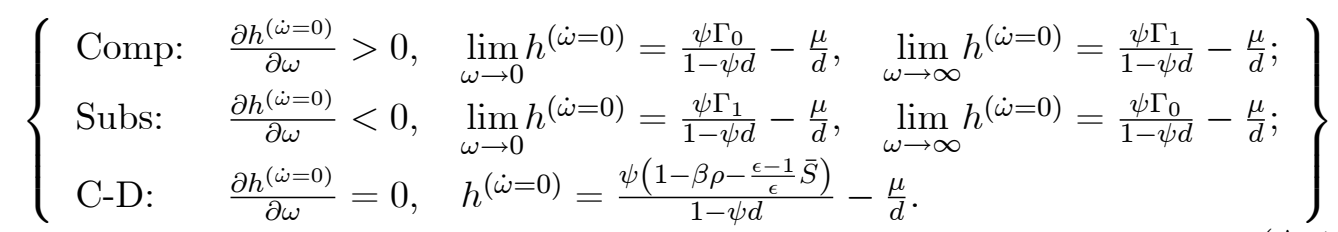

Now consider the locus $h^{(\dot{h}=0)}$. From $(24)$, we have

$$
h^{(\dot{h}=0)}=\frac{1}{\rho}\left(\sigma+\frac{1-\psi b^{*}(\omega, h)}{y^{*}(\omega, h)}\right)=\frac{1}{\rho}\left[1-\beta \rho-\frac{\epsilon-1}{\epsilon} S\left(p^{*}(\omega, h)\right)+\sigma\right],
$$

where we have used condition (A.27) evaluated in equilibrium to obtain the last term. Using the properties of $S(p)$ listed in (22) and (A.24), as well as the result $\frac{\partial}{\partial \omega} p^{*}(\omega, h)<0$ in Proposition 1 , we have

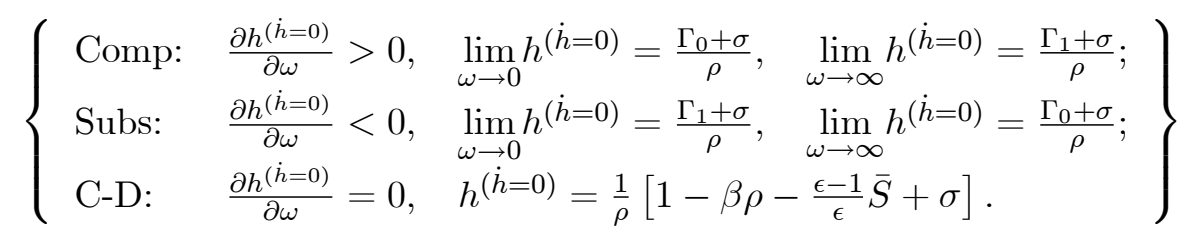

Results (A.49) and (A.51) imply that both $h^{(\dot{\omega}=0)}$ and $h^{(\dot{h}=0)}$ are increasing (decreasing) in the $(\omega, h)$ plane under complementarity (substitutability) while they are both horizontal straight lines in the Cobb-Douglas case. 
Step \#2. Denote by $\Lambda(0)$ the distance between the vertical intercepts of $h^{(\dot{\omega}=0)}$ and $h^{(\dot{h}=0)}$ and, similarly, denote by $\Lambda(\infty)$ the vertical distance between the asymptotic levels of $h^{(\dot{\omega}=0)}$ and $h^{(\dot{h}=0)}$ as $\omega \rightarrow \infty$, i.e.,

$$
\Lambda(0) \equiv \lim _{\omega \rightarrow 0} h^{(\dot{\omega}=0)}-\lim _{\omega \rightarrow 0} h^{(\dot{h}=0)} \text { and } \Lambda(\infty) \equiv \lim _{\omega \rightarrow \infty} h^{(\dot{\omega}=0)}-\lim _{\omega \rightarrow \infty} h^{(\dot{h}=0)} .
$$

First, consider the case of complementarity. From (A.49) and (A.51), we have

$$
\text { Comp: } \quad \Lambda(0)=\frac{1}{\rho}\left[\frac{\psi \rho-(1-\psi d)}{1-\psi d} \cdot \Gamma_{0}-\sigma-\mu(\rho / d)\right] .
$$

Substituting (A.45) and recalling the definition of $\Gamma_{0}$ in (A.46), the above expression reduces to

$$
\text { Comp: } \quad \Lambda(0)=-\frac{1}{\rho} \cdot \frac{\psi \rho-(1-\psi d)}{1-\psi d} \cdot \frac{\epsilon-1}{\epsilon} \cdot\left(1-S\left(p^{s s}\right)\right)<0 .
$$

Similarly, calculating $\Lambda(\infty)$ from (A.49) and (A.51), using (A.45) and (A.46) to eliminate $\Gamma_{1}$, we get

$$
\text { Comp: } \quad \Lambda(\infty)=\frac{1}{\rho} \cdot \frac{\psi \rho-(1-\psi d)}{1-\psi d} \cdot \frac{\epsilon-1}{\epsilon} \cdot S\left(p^{s s}\right)>0 .
$$

Results (A.53)-(A.54) imply that, under complementarity, $h^{(\dot{h}=0)}$ cuts $h^{(\dot{\omega}=0)}$ from above. Repeating the same steps for the case of substitutability, we obtain the symmetric results

$$
\begin{array}{ll}
\text { Subs: } & \Lambda(0)=\frac{1}{\rho} \cdot \frac{\psi \rho-(1-\psi d)}{1-\psi d} \cdot \frac{\epsilon-1}{\epsilon} \cdot S\left(p^{s s}\right)>0 \\
\text { Subs: } & \Lambda(\infty)=-\frac{1}{\rho} \cdot \frac{\psi \rho-(1-\psi d)}{1-\psi d} \cdot \frac{\epsilon-1}{\epsilon} \cdot\left(1-S\left(p^{s s}\right)\right)<0
\end{array}
$$

so that, under substitutability, $h^{(\dot{h}=0)}$ cuts $h^{(\dot{\omega}=0)}$ from below.

Step \#3. Linearizing system (23)-(24) around $\left(\omega^{s s}, h^{s s}\right)$, we obtain

$$
\left(\begin{array}{c}
\dot{\omega} \\
\dot{h}
\end{array}\right) \simeq\left(\begin{array}{ll}
m_{1} & m_{2} \\
m_{3} & m_{4}
\end{array}\right)\left(\begin{array}{c}
\left(\omega-\omega^{s s}\right) \\
\left(h-h^{s s}\right)
\end{array}\right),
$$

where the coefficient matrix is

$$
\begin{array}{ll}
m_{1} \equiv-\left.\omega^{s s} \cdot \frac{\partial b(\omega, h)}{\partial \omega}\right|_{\omega=\omega^{s s}} \quad \text { and } \quad m_{2} \equiv-\left.\omega^{s s} \cdot \frac{\partial b(\omega, h)}{\partial h}\right|_{h=h^{s s}}, \\
\left.m_{3} \equiv \frac{\epsilon-1}{\epsilon} \cdot \frac{\partial}{\partial \omega} S\left(p^{*}(\omega, h)\right)\right|_{\omega=\omega^{s s}} \quad \text { and } \quad m_{4} \equiv \rho+\left.\frac{\epsilon-1}{\epsilon} \cdot \frac{\partial}{\partial h} S\left(p^{*}(\omega, h)\right)\right|_{h=h^{s s}} .
\end{array}
$$


Denoting the characteristic roots of system (A.57) by $\tilde{\lambda}_{1,2}$, we have

$$
\tilde{\lambda}_{1,2}=\frac{\left(m_{1}+m_{4}\right) \pm \sqrt{\left(m_{1}+m_{4}\right)^{2}-4\left(m_{1} m_{4}-m_{2} m_{3}\right)}}{2} .
$$

Consider the case of substitutability. By Proposition 1, substitutability implies $m_{1}<$ $0, m_{2}<0, m_{3}>0, m_{4}>0$. Since both stationary loci are decreasing and $h^{(\dot{\omega}=0)}$ is steeper than $h^{(\dot{h}=0)}$, the linearized slopes must satisfy

$$
\text { Subs: } \quad-m_{1} / m_{2}<-m_{3} / m_{4} \text {. }
$$

Given that $m_{2}<0$ and $m_{4}>0$, inequality (A.60) implies

$$
\text { Subs: } \quad m_{1} m_{4}<m_{2} m_{3} \text {. }
$$

From (A.61), the term under the square root in (A.59) is strictly positive and greater than $\left(m_{1}+m_{4}\right)^{2}$, which implies two real roots of opposite sign - i.e., saddle-point stability. Next, consider the case of complementarity. By Proposition 1, complementarity implies $m_{1}>0, m_{2}<0, m_{3}<0$ and $m_{4}>0 .{ }^{17}$ Since both stationary loci are increasing and $h^{(\dot{\omega}=0)}$ is steeper than $h^{(\dot{h}=0)}$, the linearized slopes must satisfy

$$
\text { Comp.: } \quad-m_{1} / m_{2}>-m_{3} / m_{4} \text {. }
$$

Given that $m_{2}<0$ and $m_{4}>0$, inequality (A.62) implies

$$
\text { Comp.: } \quad m_{1} m_{4}>m_{2} m_{3} \text {. }
$$

From (A.63), the term under the square root in (A.59) is smaller than $\left(m_{1}+m_{4}\right)^{2}$, which implies that the steady state is a node (or a focus). The fact that $m_{1}+m_{4}>0$ implies that the node (focus) is unstable.

Dynamics in the Cobb-Douglas case. From (A.49) and (A.51) in the proof of Proposition 5, the stationary loci are horizontal lines and generate the three cases described in Figure 3.

Derivation of (38)-(39). Equations (38)-(39) are obtained following exactly the same steps as in the derivations of (20)-(39) using the new wealth constraint $(37)$.

\footnotetext{
${ }^{17}$ While the signs $m_{1}>0, m_{2}<0, m_{3}<0$ can be directly derived from Proposition $1, m_{4}>0$ follows from the fact that the $h^{(\dot{h}=0)}$ locus is strictly increasing: being the linearized slope equal to $-m_{3} / m_{4}$ with $m_{3}<0$, having $-m_{3} / m_{4}>0$ necessarily requires $m_{4}>0$.
} 
Proof of result (40). Treating $(s, h)$ as parameters, the system formed by (19), (38) and (39) can be rewritten as

$$
\begin{aligned}
& y_{1}(b ; h) \equiv \frac{\psi}{h+(\mu / b)}, \\
& y_{2}(b, p ; s) \equiv \frac{1-\psi b}{1-\beta \rho}+\frac{s}{1-\beta \rho} \cdot p, \\
& y_{3}(b, p) \equiv \frac{1-\psi b}{1-\beta \rho-\frac{\epsilon-1}{\epsilon} S(p)},
\end{aligned}
$$

which is identical to system (A.25)-(A.27) up to the fact that net supply per adult $s$ replaces endowment per adult $\omega$. Consequently, we can follow the same steps as in the proof of Proposition 1 to obtain analogous results. In particular, by analogy with (A.30), the model with minimum resource requirements yields

$$
\text { (all cases): } \frac{\partial \bar{p}(b ; s)}{\partial s}<0, \quad \lim _{s \rightarrow 0^{+}} \bar{p}(b ; s)=\infty, \quad \lim _{s \rightarrow \infty} \bar{p}(b ; s)=0,
$$

where the first limit in (A.65) implies result (40) in the text.

Derivation of (42). Consider the household problem in the presence of minimum resource requirement (section 6). Given the new constraint (37), the currentvalue Hamiltonian reads

$\mathcal{L}^{s} \equiv \log Y+\mu \log b+\sigma \log L+\lambda^{s} \cdot[r A+w L(1-\psi b)+p(\Omega-\eta L)-Y]+\ell^{s} \cdot[L(b-d)]$,

where $\lambda^{s}$ and $\ell^{s}$ are the dynamic multipliers. The first three necessary conditions for optimality coincide with (A.4), (A.5) and (A.6) after re-defining the multipliers accordingly. Instead, the costate equation (A.7) is now replaced by

$$
\rho \ell^{s}(t)-\dot{\ell}^{s}(t)=\frac{\sigma}{L(t)}+\lambda^{s}(t) \cdot[w(t)(1-\psi b(t))-p(t) \eta]+\ell^{s}(t) \cdot(b(t)-d) .
$$

Re-defining the shadow value of humanity as $h(t) \equiv \ell^{s}(t) L(t)$, and substituting $\lambda^{s}(t)=1 / Y(t)$ from (A.4), condition (A.66) implies

$$
\rho-\frac{\dot{h}(t)}{h(t)}=\frac{\sigma}{h(t)}+\frac{w(t)(1-\psi b(t))-p(t) \eta}{h(t) y(t)} .
$$

Setting $w(t)=1$ and rearranging terms in the above expression, we obtain (42).

Derivation of (43). Imposing $\dot{s}=\dot{h}=0$ in system (41)-(4224), we obtain

$$
b^{*}\left(s^{s s}, h^{s s}\right)=d \text { and } h^{s s}=\frac{1}{\rho} \cdot\left(\sigma+\frac{1-\psi d-\eta p^{*}\left(s^{s s}, h^{s s}\right)}{y^{*}\left(s^{s s}, h^{s s}\right)}\right) .
$$


From condition (19), in the steady state we also have

$$
h^{s s}=\frac{\psi}{y^{*}\left(s^{s s}, h^{s s}\right)}-\frac{\mu}{d} .
$$

Combining (A.67) and (A.68) to eliminate $h^{s s}$, we obtain

$$
y^{*}\left(s^{s s}, h^{s s}\right)=\frac{\psi \rho-\left[1-\psi d-\eta p^{*}\left(s^{s s}, h^{s s}\right)\right]}{\sigma+\mu(\rho / d)} .
$$

From (38), we can substitute

$$
p^{*}\left(s^{s s}, h^{s s}\right) \cdot s^{s s}=y^{*}\left(s^{s s}, h^{s s}\right) \cdot(1-\beta \rho)-(1-\psi d)
$$

into (A.69) to eliminate $p^{*}\left(s^{s s}, h^{s s}\right)$, obtaining (43).

Proof of Proposition 6. Imposing $\dot{s}=0$ in (41), the locus $h^{(\dot{s}=0)}$ is characterized by $b^{*}(s, h)=d$ and has exactly the same qualitative properties as $h^{(\dot{\omega}=0)}$ in the model with no resource requirement $(\eta=0)$. Imposing $\dot{h}=0$ in (42), the locus $h^{(\dot{h}=0)}$ in the presence of minimum resource requirement is characterized by the condition

$$
\begin{aligned}
h^{(\dot{h}=0)} & =\frac{\sigma}{\rho}+\frac{1-\psi b^{*}(s, h)-\eta \cdot p^{*}(s, h)}{\rho y^{*}(s, h)} \\
& =\frac{1}{\rho}\left\{\sigma+\frac{1-\psi b^{*}(s, h)-\eta \cdot p^{*}(s, h)}{1-\psi b^{*}(s, h)} \cdot\left[1-\beta \rho-\frac{\epsilon-1}{\epsilon} S\left(p^{*}(s, h) \gamma\right] A\right\} 7,1\right)
\end{aligned}
$$

where we have used the last condition in (A.64) to obtain the last term. Using the properties of $S(p)$ listed in (22) and (A.24) with reference to $s$ instead of $\omega$, as well as $\frac{\partial}{\partial s} p^{*}(s, h)<0$ by analogy with Proposition 1 , we have

$$
\left\{\begin{array}{llll}
\text { Comp: } & \frac{\partial h^{(\dot{h}=0)}}{\partial s}>0, & \lim _{s \rightarrow 0} h^{(\dot{h}=0)}=-\infty, & \lim _{s \rightarrow \infty} h^{(\dot{h}=0)}=\frac{\Gamma_{1}+\mu-1}{\rho} ; \\
\text { Subs: } & \frac{\partial h^{(h=0)}}{\partial s} \gtrless 0 & \lim _{s \rightarrow 0} h^{(\dot{h}=0)}=-\infty, & \lim _{s \rightarrow \infty} h^{(\dot{h}=0)}=\frac{\Gamma_{0}+\mu-1}{\rho} ; \\
\text { C-D: } & \frac{\partial h^{(\dot{h}=0)}}{\partial s}>0, & \lim _{s \rightarrow 0} h^{(\dot{h}=0)}=-\infty, & \lim _{s \rightarrow \infty} h^{(\dot{h}=0)}=\frac{1}{\rho}\left[1-\beta \rho-\frac{\epsilon-1}{\epsilon} \bar{S}+\mu-1\right] .
\end{array}\right\},
$$

where the sign of $\frac{\partial h^{(\dot{h}=0)}}{\partial s}$ under substitutability is positive for $s$ close to zero (the price effect dominates) whereas it becomes zero and then strictly negative as $s$ assumes higher values (the fertility/income effect dominates as in the model with $\eta=0$ ). These results determine the phase diagrams reported in Figure 4. By analogy with the proof of Proposition 5, the stability (instability) of the various steady states is determined by the fact that $h^{(\dot{h}=0)}$ cuts $h^{(\dot{s}=0)}$ from below (above). 